\title{
On nonadiabatic contributions to the neutrino oscillation probability and the formalism by Kimura, Takamura and Yokomakura
}

\author{
Osamu Yasuda \\ Department of Physics, Tokyo Metropolitan University, \\ Minami-Osawa, Hachioji, Tokyo 192-0397, Japan
}

\begin{abstract}
It is shown that it is possible to obtain the analytical expression for the effective mixing angle in matter using the formalism which was developed by Kimura, Takamura and Yokomakura for the neutrino oscillation probability in matter with constant density. If we assume that the imaginary part of the integral of the difference of the energy eigenvalues of the two levels at each level-crossing is given by the ratio $\gamma$ of the difference of the energy eigenvalues of the two levels to the derivative of the effective mixing angle at the level-crossing, then the nonadiabatic contribution to the oscillation probability can be expressed analytically by this formalism. We give one example in which the energy eigenvalues cannot be expressed as roots of a quadratic equation and we show that our assumption is correct in the approximation of the small mixing angle.
\end{abstract}

PACS numbers: 14.60.Pq, 14.60.St 


\section{INTRODUCTION}

Since the discovery of oscillation of atmospheric neutrinos [1], neutrino oscillation has attracted a lot of attention. To discuss the behaviors of neutrino oscillation intuitively, it is important to have analytical formulae for the oscillation probability. However, it is difficult to obtain an analytical formula in the three flavor mixing scheme in matter. In 2002 Kimura, Takamura and Yokomakura (KTY) discovered a compact formula [2, 3] for the neutrino oscillation probability in matter with constant density. Subsequently the KTY framework was generalized to more general cases. Ref. [4] discussed the four neutrino mixing scheme in matter with constant density. Ref. [5] discussed two cases of neutrino oscillation in the adiabatic approximation, the one with non-standard interactions where the matter potential has non-diagonal elements in the flavor basis, or the other with large neutrino magnetic moments in a magnetic field.

In general, however, adiabatic approximation may not be good, and in the present paper we discuss nonadiabatic contributions to the oscillation probability. It is believed ${ }^{1}$ that the nonadiabatic contributions to the transition phenomena in a problem with three or more eigenstates can be treated approximately well by applying the method for two state problems [6, 7] at each level-crossing, if the the two resonances are sufficiently far apart.

In the two flavor case, nonadiabatic contributions to the oscillation probability is approximately obtained by the WKB method [6] in which the imaginary part of the integral of the difference of the energy eigenvalues of the two levels is evaluated. The imaginary part of this integral is proportional to the ratio $\gamma$ of the difference of the energy eigenvalues of the two levels to the derivative of the effective mixing angle at the level-crossing. In the three flavor case with standard matter effect, the situation at each level-crossing is essentially the same as that in the two flavor case, because the energy eigenvalues are the roots of quadratic equations and the procedure for diagonalization is the same as that for the two flavor case. So one can discuss analytically the oscillation probability of supernova neutrinos ${ }^{2}$, which are supposed to go through the two level-crossing points.

In general case of neutrino oscillation with nonstandard matter effects or with more than three flavors, however, it is nontrivial to obtain the analytical expression for the energy eigenvalues and the effective mixing angles. In this paper we show that, if the energy eigenvalues are obtained analytically, then it is possible to obtain the analytical expression for the effective mixing angle using the KTY formalism. Furthermore, assuming that the imaginary part of the integral of the difference of the energy eigenvalues of the two levels at each level-crossing is given by the ratio $\gamma$ of the difference of the energy eigenvalues of the two levels to the derivative of the effective mixing angle at the level-crossing, we argue that nonadiabatic contributions to the oscillation probability can be expressed analytically by the KTY formalism. We give one example in which the energy eigenvalues cannot be expressed as roots of a quadratic equation and we show that our assumption is correct in the small mixing angle limit.

Throughout this paper we discuss the case in which the baseline of the neutrino path is

${ }^{1}$ See, e.g., Ref. [8] and references therein. See also Ref. [9] for a discussion on the condition to justify such a treatment.

2 It was pointed out that so-called collective oscillations [10, 11] could be important for phenomenology of supernova neutrinos. However, this topics is beyond the scope of paper, and we do not discuss the effect of collective oscillations here. 
long enough so that averaging over rapid oscillations is a good approximation, as in the case of the solar neutrino deficit phenomena. We also assume normal hierarchy for simplicity. The case with inverted hierarchy can be treated by the same manner.

The paper is organized as follows. In Sec. II, we review basic results of neutrino oscillation in the adiabatic approximation. In Sec. III, we discuss the nonadiabatic contributions to the flavor transition, and show how to express the effective mixing angle using the KTY formalism. In Sec. IV, we apply our general idea of Sec. III to two examples. In Sec. V, we draw our conclusions. In the appendices A, B, C, D, E, F, G, and H we provide details of the derivation of the analytic formulae for the energy eigenvalues, the effective mixing angles and the nonadiabatic contributions to oscillation probabilities.

\section{THE OSCILLATION PROBABILITY IN THE ADIABATIC APPROXIMA- TION AND THE EFFECTIVE MIXING ANGLES IN MATTER}

\section{A. The oscillation probability in matter}

The equation of motion for neutrinos propagating in matter with general potential is given by

$$
i \frac{d \Psi}{d t}=\left[U \mathcal{E}_{0} U^{-1}+\mathcal{A}(t)\right] \Psi
$$

where

$$
\begin{aligned}
\mathcal{E}_{0} & \equiv \operatorname{diag}\left(E_{1}, E_{2}, E_{3}\right) \\
\mathcal{A}(t) & \equiv\left(\begin{array}{lll}
A_{e e}(t) & A_{e \mu}(t) & A_{e \tau}(t) \\
A_{\mu e}(t) & A_{\mu \mu}(t) & A_{\mu \tau}(t) \\
A_{\tau e}(t) & A_{* \mu}(t) & A_{\tau \tau}(t)
\end{array}\right) .
\end{aligned}
$$

$\Psi^{T} \equiv\left(\nu_{e}, \nu_{\mu}, \nu_{\tau}\right)$ is the flavor eigenstate, $U$ is the leptonic mixing matrix, $E_{j} \equiv$

$\sqrt{m_{j}^{2}+\vec{p}^{2}}(j=1,2,3)$ is the energy eigenvalue of each mass eigenstate, and the matter effect $\mathcal{A}(t)$ at time (or position ) $t$ is characterized by the matter potential $A_{\alpha \beta}(t)(\alpha, \beta=e, \mu, \tau)$. Since the matrix which is proportional to identity gives contribution only to the phase of the probability amplitude, instead of $\mathcal{E}_{0}$ itself, we use the following quantity:

$$
\mathcal{E} \equiv \mathcal{E}_{0}-E_{1} \mathbf{1}=\operatorname{diag}\left(0, \Delta E_{21}, \Delta E_{31}\right)
$$

where

$$
\Delta E_{j k} \equiv E_{j}-E_{k} \simeq\left(m_{j}^{2}-m_{k}^{2}\right) / 2|\vec{p}|
$$

The $3 \times 3$ matrix on the right hand side of Eq. (1) can be formally diagonalized as:

$$
U \mathcal{E} U^{-1}+\mathcal{A}(t)=\tilde{U}(t) \tilde{\mathcal{E}}(t) \tilde{U}^{-1}(t)
$$

where

$$
\tilde{\mathcal{E}}(t) \equiv \operatorname{diag}\left(\tilde{E}_{1}(t), \tilde{E}_{2}(t), \tilde{E}_{3}(t)\right)
$$


is a diagonal matrix with the energy eigenvalues $\tilde{E}_{j}(t)$ in the presence of the matter effect.

In this section we consider the case where the density of the matter varies adiabatically as in the case of the solar neutrino deficit phenomena. In this case, we get

$$
\Psi(L)=\tilde{U}(L) \exp \left[-i \int_{0}^{L} \tilde{\mathcal{E}}(t) d t\right] \tilde{U}(0)^{-1} \Psi(0)
$$

where $\tilde{U}(0)$ and $\tilde{U}(L)$ stand for the effective mixing matrices at the origin $t=0$ and at the endpoint $t=L$. The oscillation probability is given by

$$
P\left(\nu_{\alpha} \rightarrow \nu_{\beta}\right)=\sum_{j, k} \tilde{U}_{\beta j}(L) \tilde{U}_{\beta k}^{*}(L) \tilde{U}_{\alpha j}^{*}(0) \tilde{U}_{\alpha k}(0) \exp \left[-i \int_{0}^{L} \Delta \tilde{E}_{j k}(t) d t\right]
$$

where we have defined

$$
\Delta \tilde{E}_{j k}(t) \equiv \tilde{E}_{j}(t)-\tilde{E}_{k}(t)
$$

Eq. (44) requires the quantity $\tilde{U}_{\beta j}(t) \tilde{U}^{*}(t)_{\beta k}$ which has the same flavor index $\beta$ but different mass eigenstate indices $j, k$, and it turns out that the analytical expression for $\tilde{U}_{\beta j}(t) \tilde{U}^{*}(t)_{\beta k}$ is hard to obtain. However, if the length $L$ of the neutrino path is very large and if $\left|\int_{0}^{L} \Delta \tilde{E}(t)_{j k} d t\right| \gg 1$ is satisfied for $j \neq k$, then, after averaging over rapid oscillations as in the case of the solar neutrino deficit phenomena, Eq. (4) is reduced to

$$
P\left(\nu_{\alpha} \rightarrow \nu_{\beta}\right)=\sum_{j} \tilde{X}_{j}^{\beta \beta}(L) \tilde{X}_{j}^{\alpha \alpha}(0)=\sum_{j} X_{j}^{\beta \beta} \tilde{X}_{j}^{\alpha \alpha}(0)
$$

where we have defined

$$
\begin{aligned}
\tilde{X}_{j}^{\alpha \beta}(t) & \equiv \tilde{U}_{\alpha j}(t) \tilde{U}_{\alpha j}(t)^{*} \\
\tilde{X}_{j}^{\alpha \alpha}(t) & \equiv\left|\tilde{U}_{\alpha j}(t)\right|^{2}
\end{aligned}
$$

and here and in the following we assume that there is no matter at the end of the baseline $t=L$.

It is known [2, 3, 5, 13] that the quantity $\tilde{X}_{j}^{\alpha \beta}(t) \equiv \tilde{U}_{\alpha j}(t) \tilde{U}_{\alpha j}(t)^{*}$ can be expressed as

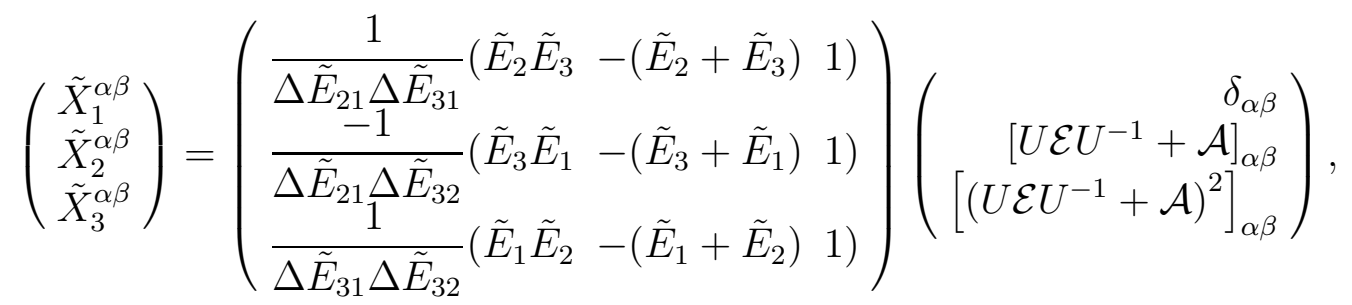

where the $t$-dependence of the quantities $\tilde{X}_{j}^{\alpha \beta}, \tilde{E}_{j}, \Delta \tilde{E}_{j k}$ is suppressed for simplicity in Eq. (7). $\left[\left(U \mathcal{E} U^{-1}+\mathcal{A}\right)^{j}\right]_{\alpha \beta}(j=1,2)$ on the right hand side are given by the known quantities although the computations are tedious for general potential $\mathcal{A}(t)$. 


\section{THE NONADIABATIC CORRECTIONS TO THE OSCILLATION PROBA- BILITY}

\section{A. The the standard case}

When adiabatic approximation is not good, Eq. (5) should be modified by taking nonadiabatic contributions into account. Substituting the diagonalized form (2) of the Hamiltonian into the Dirac equation (1), we have

$$
i \frac{d}{d t}\left\{\tilde{U}^{-1}(t) \Psi(t)\right\}=\left[\tilde{\mathcal{E}}-i \tilde{U}^{-1}\left(\frac{d}{d t} \tilde{U}\right)\right]\left\{\tilde{U}^{-1}(t) \Psi(t)\right\},
$$

where $\tilde{U}^{-1}(t) \Psi(t)$ is the effective energy eigenstate.

In the two flavor case, there is only one level crossing, and the probability $P\left(\nu_{\alpha} \rightarrow \nu_{\beta}\right)$ is given by

$$
P\left(\nu_{\alpha} \rightarrow \nu_{\beta}\right)=\left(\begin{array}{ll}
\left|U_{\beta 1}\right|^{2} & \left|U_{\beta 2}\right|^{2}
\end{array}\right)\left(\begin{array}{cc}
1-P_{C} & P_{C} \\
P_{C} & 1-P_{C}
\end{array}\right)\left(\left.\begin{array}{c}
\mid \tilde{U}_{\alpha 1}(0) \\
\mid \tilde{U}_{\alpha 2}(0)
\end{array}\right|^{2}\right)
$$

where $P_{C}$ stands for the jumping probability from the energy eigenstate $\tilde{\nu}_{1}$ to $\tilde{\nu}_{2}$, and is approximately computed by the WKB method [6] :

$$
P_{C}=\exp \left[-\operatorname{Im} \int_{C} \Delta \tilde{E}(t) d t\right]
$$

Here $\Delta \tilde{E}(t)$ is the difference of the two energy eigenvalues at the level crossing, and the contour $C$ is defined as a path from $t=t_{0}$ to $t=t_{1}$ where $t_{0}$ is a point which gives the minimum value of $\Delta \tilde{E}(t)$, and $t_{1}$ is a point in the complex $x$-plane such that $\Delta \tilde{E}\left(t_{1}\right)=0$. Throughout this paper we assume that the WKB approximation (9) is good ${ }^{3}$. It is known that the exponent in (9) is related by the ratio $\gamma$ of the difference of the energy eigenvalues of the two levels to the derivative of the effective mixing angle $\tilde{\theta}$ at the level-crossing:

$$
-\log P_{C}=\frac{\pi}{2} F \gamma
$$

where

$$
\gamma=\left.\frac{\Delta \tilde{E}}{2|d \tilde{\theta} / d t|}\right|_{\text {resonance }}
$$

The subscript resonance stands for the quantity evaluated at the point where the difference $|\Delta \tilde{E}|$ of the two energy eigenvalues becomes minimum. $F$ is the factor which depends on

3 In the extreme nonadiabatic limit, Eq. (9) need a certain modification [16]. However, the major purpose of this paper is to show how to handle nonadiabatic corrections in the case with more than two flavors, rather than computing the deviation from the linear potential or the corrections due to the extreme nonadiabatic condition. These corrections can be treated in the same manner as in the two flavor case, so we do not include such a modification for simplicity in this paper. 
the form of the potential $A$, and in the case of linear potential, i.e., in the case $A \propto t$ we have $F=1^{4}$. In the standard two flavor case, we have

$$
\gamma=\frac{\Delta E_{21} \sin ^{2} 2 \theta}{\cos 2 \theta|d \log A / d t|_{\text {resonance }}}
$$

In the three flavor case, there are at most two level crossings for neutrinos as in the case of a supernova [12]. Assuming that the nonadiabatic transition at each level crossing can be described by the same manner as in the case of the two level-crossing, and assuming that the level crossing occurs at $\tilde{E}_{3} \simeq \tilde{E}_{1}$ and $\tilde{E}_{2} \simeq \tilde{E}_{1}$, the transition probability is given by

$$
\begin{aligned}
P\left(\nu_{\alpha} \rightarrow \nu_{\beta}\right)= & \left(\left|U_{\beta 1}\right|^{2}\left|U_{\beta 2}\right|^{2}\left|U_{\beta 3}\right|^{2}\right)\left(\begin{array}{ccc}
1-P_{L} & P_{L} & 0 \\
P_{L} & 1-P_{L} & 0 \\
0 & 0 & 1
\end{array}\right) \\
& \times\left(\begin{array}{ccc}
1-P_{H} & 0 & P_{H} \\
0 & 1 & 0 \\
P_{H} & 0 & 1-P_{H}
\end{array}\right)\left(\begin{array}{c}
\left|\tilde{U}_{\alpha 1}(0)\right|^{2} \\
\left|\tilde{U}_{\alpha 2}(0)\right|^{2} \\
\left|\tilde{U}_{\alpha 3}(0)\right|^{2}
\end{array}\right) .
\end{aligned}
$$

Using the WKB approximation [6], the jumping factors in (11) are given by

$$
\begin{aligned}
P_{H} & =\exp \left[-\operatorname{Im} \int_{C} \Delta \tilde{E}_{31}(t) d t\right], \\
P_{L} & =\exp \left[-\operatorname{Im} \int_{C} \Delta \tilde{E}_{21}(t) d t\right] .
\end{aligned}
$$

As is shown in Appendix A,

$$
\tilde{\theta}_{13} \equiv \frac{1}{2} \tan ^{-1} \frac{\Delta E_{31} \sin 2 \theta_{13}}{\Delta E_{31} \cos 2 \theta_{13}-A}
$$

is the effective mixing angle near the level-crossing $\Delta E_{31} \simeq A$, while

$$
\tilde{\theta}_{12} \equiv \frac{1}{2} \tan ^{-1} \frac{\Delta E_{21} \sin 2 \theta_{12}}{\Delta E_{21} \cos 2 \theta_{12}-A c_{13}^{2}}
$$

is the effective mixing angle near the level-crossing $\Delta E_{21} \simeq A$. As in the case of a two level problem, we have the following jumping probabilities:

$$
\begin{aligned}
P_{H} & =\exp \left(-\frac{\pi}{2} F \frac{\Delta E_{31} \sin ^{2} 2 \theta_{13}}{\cos 2 \theta_{13}|d \log A / d t|_{\text {resonance }}}\right), \\
P_{L} & =\exp \left(-\frac{\pi}{2} F \frac{\Delta E_{21} \sin ^{2} 2 \theta_{12}}{\cos 2 \theta_{12}|d \log A / d t|_{\text {resonance }}}\right) .
\end{aligned}
$$

$F$ is the factor which depends on the form of the potential $A$, and $F=1$ in the case of a linear potential.

${ }_{4} F$ can be evaluated analytically for a few cases of the density profile such as $t, 1 / t$ and $e^{-t}$. See Ref. [8] and references therein. 
The effective mixing matrix elements $\left|\tilde{U}_{\alpha j}(0)\right|^{2}$ at the origin $L=0$ can be approximately obtained by putting $\Delta m_{21}^{2} \rightarrow 0$ and by substituting $\theta_{12} \rightarrow 0, \theta_{13} \rightarrow \tilde{\theta}_{13}, \delta \rightarrow 0$. They are thus given by

$$
\left|\tilde{U}_{\alpha j}(0)\right|^{2}=\left(\begin{array}{ccc}
\tilde{c}_{13}^{2} & 0 & \tilde{s}_{13}^{2} \\
\tilde{s}_{13}^{2} s_{23}^{2} & c_{23}^{2} & \tilde{c}_{13}^{2} s_{23}^{2} \\
\tilde{s}_{13}^{2} c_{23}^{2} & s_{23}^{2} & \tilde{c}_{13}^{2} c_{23}^{2}
\end{array}\right)
$$

where $\tilde{c}_{13} \equiv \cos \tilde{\theta}_{13}$ and $\tilde{s}_{13} \equiv \sin \tilde{\theta}_{13}$ are the quantities which are evaluated from Eq. (12) at the origin $L=0$. From Eqs. (11), (13), (14) and (15), we have the analytical expression for the transition probability including the nonadiabatic contributions in the three flavor standard case.

\section{B. The effective mixing angles in matter}

The energy eigenvalues in the previous discussions are easily obtained because the eigenvalues near each level-crossing in the standard case are the roots of a quadratic equation in the leading order in $\Delta E_{21} / \Delta E_{31}$. In some cases with more than two states, however, the energy eigenvalues cannot be expressed as the roots of a quadratic equation, and in that case it is difficult to compute the jumping factor (9). Assuming that the equality (10) holds at each level-crossing, it is useful to define the effective mixing angle in the presence of the matter potential $\mathcal{A}(t)^{5}$.

In this subsection we will show how to derive the expression for the effective mixing angle in the presence of the matter using the KTY formalism. The KTY formalism enables one to obtain the bilinear quantity $\tilde{X}_{j}^{\alpha \beta}$ defined in Eq. (6). Our strategy here is to start with effective matrix elements $\tilde{X}_{j}^{\alpha \beta}$ which are obtained by the KTY formalism and to determine the phase of each element by demanding that it be consistent with the standard form (B1) of the mixing matrix element in vacuum. We will discuss only the three flavor case, but we can generalize this method to the case with more than three flavors.

From the identities

$$
\begin{aligned}
\tilde{U}_{e j} & =\sqrt{\tilde{X}_{j}^{e e}} e^{i \arg \tilde{U}_{e j}}, \\
\tilde{U}_{\mu j} & =\frac{\tilde{X}_{j}^{\mu e}}{\sqrt{\tilde{X}_{j}^{e e}}} e^{i \arg \tilde{U}_{e j}}, \\
\tilde{U}_{\tau j} & =\frac{\tilde{X}_{j}^{\tau e}}{\sqrt{\tilde{X}_{j}^{e e}}} e^{i \arg \tilde{U}_{e j}}
\end{aligned}
$$

the first guess for the effective mixing matrix $\tilde{U}$ can be written as

$$
\tilde{U}_{1}=\tilde{U}_{0} \times \operatorname{diag}\left(e^{i \arg \tilde{U}_{e 1}}, e^{i \arg \tilde{U}_{e 2}}, e^{i \arg \tilde{U}_{e 3}}\right),
$$

\footnotetext{
5 The effective mixing angles were given in the standard three flavor case in Ref. 14]
} 
where

$$
\tilde{U}_{0} \equiv\left(\left(\begin{array}{c}
\sqrt{\tilde{X}_{1}^{e e}} \\
\tilde{X}_{1}^{\mu e} / \sqrt{\tilde{X}_{1}^{e e}} \\
\tilde{X}_{1}^{\tau e} / \sqrt{\tilde{X}_{1}^{e e}}
\end{array}\right),\left(\begin{array}{c}
\sqrt{\tilde{X}_{2}^{e e}} \\
\tilde{X}_{2}^{\mu e} / \sqrt{\tilde{X}_{2}^{e e}} \\
\tilde{X}_{2}^{\tau e} / \sqrt{\tilde{X}_{2}^{e e}}
\end{array}\right),\left(\begin{array}{c}
\sqrt{\tilde{X}_{3}^{e e}} \\
\tilde{X}_{3}^{\mu e} / \sqrt{\tilde{X}_{3}^{e e}} \\
\tilde{X}_{3}^{\tau e} / \sqrt{\tilde{X}_{3}^{e e}}
\end{array}\right)\right) .
$$

However, this naive choice is not exactly the same as the standard parametrization (B1) for the vacuum mixing matrix, because Eq. (B1) implies that $\operatorname{Im}\left(\tilde{U}_{e 1}\right)=\operatorname{Im}\left(\tilde{U}_{e 2}\right)=$ $\operatorname{Im}\left(\tilde{U}_{\mu 3}\right)=\operatorname{Im}\left(\tilde{U}_{\tau 3}\right)=0$ and $\operatorname{det} \tilde{U}=1$. In order for $\tilde{U}_{\alpha j}$ to have the expression consistent with the form (B1), therefore, we postulate the following conditions:

$$
\begin{aligned}
\arg \tilde{U}_{e 1} & =0, \\
\arg \tilde{U}_{e 2} & =0, \\
\arg \tilde{U}_{\mu 3} & =0, \\
\arg \tilde{U}_{\tau 3} & =0, \\
\arg \operatorname{det} \tilde{U}_{\alpha j} & =0 .
\end{aligned}
$$

To satisfy Eqs. (18)-(22), we multiply diagonal matrices with elements with complex phases both from the left and right hand sides of $\tilde{U}_{0}{ }^{6}$ :

$$
\tilde{U} \equiv e^{i \varphi_{0}} e^{i \varphi_{3} \lambda_{3}} e^{i \varphi_{9} \lambda_{9}} \tilde{U}_{0} e^{i \varphi_{9}^{\prime} \lambda_{9}} e^{i \varphi_{3}^{\prime} \lambda_{3}},
$$

where

$$
\begin{aligned}
& \lambda_{3} \equiv \operatorname{diag}(1,-1,0), \\
& \lambda_{9} \equiv \operatorname{diag}(1,0,-1) .
\end{aligned}
$$

It is straightforward to obtain $\varphi_{0}, \varphi_{3}, \varphi_{9}, \varphi_{3}^{\prime}, \varphi_{9}^{\prime}$ from Eqs. (18)-(22), and we get

$$
\begin{aligned}
\varphi_{0} & =-\frac{1}{3} \arg \operatorname{det} \tilde{U}_{0}, \\
\varphi_{3} & =\frac{1}{3} \arg \operatorname{det} \tilde{U}_{0}-\frac{1}{3} \arg \tilde{X}_{1}^{\mu e}+\frac{1}{3} \arg \tilde{X}_{3}^{\mu e}-\frac{2}{3} \arg \tilde{X}_{3}^{\tau e}, \\
\varphi_{9} & =\frac{1}{3} \arg \operatorname{det} \tilde{U}_{0}-\frac{1}{3} \arg \tilde{X}_{1}^{\mu e}-\frac{2}{3} \arg \tilde{X}_{3}^{\mu e}+\frac{1}{3} \arg \tilde{X}_{3}^{\tau e}, \\
\varphi_{3}^{\prime} & =\frac{1}{3} \arg \operatorname{det} \tilde{U}_{0}+\frac{1}{3} \arg \tilde{X}_{1}^{\mu e}-\frac{1}{3} \arg \tilde{X}_{3}^{\mu e}-\frac{1}{3} \arg \tilde{X}_{3}^{\tau e}, \\
\varphi_{9}^{\prime} & =-\frac{2}{3} \arg \operatorname{det} \tilde{U}_{0}+\frac{1}{3} \arg \tilde{X}_{1}^{\mu e}+\frac{2}{3} \arg \tilde{X}_{3}^{\mu e}+\frac{2}{3} \arg \tilde{X}_{3}^{\tau e} .
\end{aligned}
$$

With the values in Eqs. (26) -(30), $\tilde{U}$ in Eq. (23) has the same parametrization as that for the standard one (B1):

$$
\tilde{U}=e^{i \tilde{\theta}_{23} \lambda_{7}} \Gamma_{\tilde{\delta}}^{(13)} e^{i \tilde{\theta}_{13} \lambda_{5}}\left(\Gamma_{\tilde{\delta}}^{(13)}\right)^{-1} e^{i \tilde{\theta}_{12} \lambda_{2}},
$$

6 Since we are using all the available degrees of freedom of the phases in the $3 \times 3$ matrix, it does not matter whether we start with $\tilde{U}_{0}$ or $\tilde{U}_{1}$. For simplicity we start with $\tilde{U}_{0}$ here. 
where $\lambda_{j}(j=2,5,7)$ are the Gell-Mann matrices and defined by

$$
\begin{aligned}
\lambda_{2} & \equiv\left(\begin{array}{ccc}
0 & -i & 0 \\
i & 0 & 0 \\
0 & 0 & 0
\end{array}\right), \\
\lambda_{5} & \equiv\left(\begin{array}{ccc}
0 & 0 & -i \\
0 & 0 & 0 \\
i & 0 & 0
\end{array}\right), \\
\lambda_{7} & \equiv\left(\begin{array}{ccc}
0 & 0 & 0 \\
0 & 0 & -i \\
0 & i & 0
\end{array}\right) .
\end{aligned}
$$

Comparing Eqs. (17), (23) and (31), we find

$$
\begin{aligned}
\cos 2 \tilde{\theta}_{12} & =\frac{\tilde{X}_{1}^{e e}-\tilde{X}_{2}^{e e}}{\tilde{X}_{1}^{e e}+\tilde{X}_{2}^{e e}}, \\
\cos 2 \tilde{\theta}_{13} & =1-2 \tilde{X}_{3}^{e e} \\
\cos 2 \tilde{\theta}_{23} & =\frac{\left|\tilde{X}_{3}^{\tau e}\right|^{2}-\left|\tilde{X}_{3}^{\mu e}\right|^{2}}{\left|\tilde{X}_{3}^{\tau e}\right|^{2}+\left|\tilde{X}_{3}^{\mu e}\right|^{2}}, \\
\tilde{\delta} & =-\left(\varphi_{0}+\varphi_{3}+\varphi_{9}-\varphi_{9}^{\prime}\right)=-\arg \operatorname{det} \tilde{U}_{0}+\arg \tilde{X}_{1}^{\mu e}+\arg \tilde{X}_{3}^{\mu e}+\arg \tilde{X}_{3}^{\tau e} .
\end{aligned}
$$

Eqs. (35)-(38) are one of the new results of the present paper. Notice that the quantities $\tilde{X}_{j}^{\alpha \beta}$ and $\operatorname{det} \tilde{U}_{0}$ in Eqs. (35)-(38) are expressed in closed form by the known variables as is seen in Eq. (7) on the assumption that analytical expressions for all the eigenvalues are known.

A remark is in order. The standard parametrization (B1) is not the only one for $3 \times 3$ unitary matrices, and other parametrizations are possible as is described in Appendix B. In the three flavor case, there can be at most two level-crossings. Depending on which pair of the energy eigenvalues gets close at each level-crossing, the relevant effective mixing angle varies. The appropriate parametrization is the one in which the orthogonal matrix, which mixes the two energy eigenstates, is located on the most right-hand side of the unitary matrix $U$, because in such a parametrization the diagonalized matrix looks like $\cdots O\left(\tilde{\theta}_{j k}\right) \operatorname{diag}\left(\cdots, \tilde{E}_{j}, \cdots, \tilde{E}_{k}, \cdots\right) O\left(\tilde{\theta}_{j k}\right)^{T} \cdots$, and it becomes clear that $\tilde{\theta}_{j k}$ in the orthogonal matrix $O\left(\tilde{\theta}_{j k}\right)$ plays a role of the effective mixing angle which mixes the energy eigenstates with the energy $\tilde{E}_{j}$ and $\tilde{E}_{k}$. Furthermore in order for the effective mixing angle $\tilde{\theta}_{j k}$ to be consistent with the two flavor description, $\tilde{\theta}_{j k}$ should become maximal at the level-crossing.

\section{TWO EXAMPLES}

We can apply the general discussions in sect III to concrete examples. In this section we will discuss two examples. ${ }^{7}$ The first one is the case with non-standard interactions where the matter potential $\mathcal{A}$ has the same form as that of the standard case in some basis. The second example is the one with magnetic moments in which the energy eigenvalues cannot be expressed as roots of a quadratic equation.

7 These were discussed in Ref. [5] in the adiabatic approximation. 


\section{A. The case with non-standard interactions}

The first example is the oscillation probability in the presence of new physics in propagation [17 19]. In this case the mass matrix is given by

$$
U \mathcal{E} U^{-1}+\mathcal{A}_{N P}
$$

where

$$
\mathcal{A}_{N P} \equiv \sqrt{2} G_{F} N_{e}\left(\begin{array}{ccc}
1+\epsilon_{e e} & \epsilon_{e \mu} & \epsilon_{e \tau} \\
\epsilon_{e \mu}^{*} & \epsilon_{\mu \mu} & \epsilon_{\mu \tau} \\
\epsilon_{e \tau}^{*} & \epsilon_{\mu \tau}^{*} & \epsilon_{\tau \tau}
\end{array}\right)
$$

The dimensionless quantities $\epsilon_{\alpha \beta}$ stand for possible deviation from the standard matter effect. It is known [20] that the constraints on the parameters $\epsilon_{e \mu}, \epsilon_{\mu \mu}, \epsilon_{\mu \tau}$ are strong $\left(\left|\epsilon_{\alpha \mu}\right| \simeq\right.$ $\mathcal{O}\left(10^{-2}\right)(\alpha=e, \mu, \tau)$ while those on the parameters $\epsilon_{e e}, \epsilon_{e \tau}, \epsilon_{\tau \tau}$ are weak $\left(\left|\epsilon_{e e}\right|,\left|\epsilon_{e \tau}\right|,\left|\epsilon_{\tau \tau}\right| \simeq\right.$ $\mathcal{O}(1)$. In Ref. [21] it was found that large values $(\sim \mathcal{O}(1))$ of the parameters $\epsilon_{e e}, \epsilon_{e \tau}, \epsilon_{\tau \tau}$ are consistent with all the experimental data including those of the atmospheric neutrino data, provided that one of the eigenvalues of the matrix (39) at high energy limit becomes zero, and that such a constraint implies the relation $\epsilon_{\tau \tau} \simeq\left|\epsilon_{e \tau}\right|^{2} /\left(1+\epsilon_{e e}\right)$. For simplicity, therefore, we consider the potential matrix

$$
\mathcal{A}_{N P}=A\left(\begin{array}{clc}
1+\epsilon_{e e} & 0 & \epsilon_{e \tau} \\
0 & 0 & 0 \\
\epsilon_{e \tau}^{*} & 0 & \left|\epsilon_{e \tau}\right|^{2} /\left(1+\epsilon_{e e}\right)
\end{array}\right) .
$$

Then $\mathcal{A}_{N P}$ can be diagonalized as

$$
\mathcal{A}_{N P}=e^{i \gamma^{\prime} \lambda_{9}} e^{-i \beta \lambda_{5}} \operatorname{diag}\left(\lambda_{e^{\prime}}, 0,0\right) e^{i \beta \lambda_{5}} e^{-i \gamma^{\prime} \lambda_{9}},
$$

where

$$
\begin{aligned}
\tan \beta & =\frac{\left|\epsilon_{e \tau}\right|}{1+\epsilon_{e e}} \\
\gamma^{\prime} & \equiv \frac{1}{2} \arg \left(\epsilon_{e \tau}\right) \\
\lambda_{e^{\prime}} & =\frac{A\left(1+\epsilon_{e e}\right)}{\cos ^{2} \beta} .
\end{aligned}
$$

As is shown in Appendix C, the mass matrix (39) can be written as

$$
\begin{aligned}
& U \mathcal{E} U^{-1}+\mathcal{A}_{N P} \\
= & e^{i \gamma^{\prime} \lambda_{9}} e^{-i \beta \lambda_{5}} e^{-i \phi_{9} \lambda_{9}} e^{-i \phi_{3} \lambda_{3}}\left[U^{\prime \prime} \mathcal{E} U^{\prime \prime-1}+\operatorname{diag}\left(\lambda_{e^{\prime}}, 0,0\right)\right] e^{-i \omega_{3} \lambda_{3}} e^{-i \omega_{9} \lambda_{9}} e^{i \beta \lambda_{5}} e^{-i \gamma^{\prime} \lambda_{9}},
\end{aligned}
$$

where the phases $\phi_{3}, \phi_{9}, \omega_{3}$ and $\omega_{9}$, which are defined in Appendix $\mathbb{C}$, are introduced to make $U^{\prime \prime}$ consistent with the standard parametrization (B1). The mixing angles $\theta_{j k}^{\prime \prime}$ and the $\mathrm{CP}$ phase $\delta^{\prime \prime}$ in the standard parametrization of $U^{\prime \prime}$ are defined by

$$
\begin{aligned}
\theta_{12}^{\prime \prime}= & \tan ^{-1}\left(\left|c_{\beta} e^{-i \gamma^{\prime}} U_{e 2}+s_{\beta} e^{i \gamma^{\prime}} U_{\tau 2}\right| /\left|c_{\beta} e^{-i \gamma^{\prime}} U_{e 1}+s_{\beta} e^{i \gamma^{\prime}} U_{\tau 1}\right|\right), \\
\theta_{13}^{\prime \prime}= & \sin ^{-1}\left|c_{\beta} e^{-i \gamma^{\prime}} U_{e 3}+s_{\beta} e^{i \gamma^{\prime}} U_{\tau 3}\right|, \\
\theta_{23}^{\prime \prime}= & \tan ^{-1}\left(U_{\mu 3} /\left|c_{\beta} e^{-i \gamma^{\prime}} U_{\tau 3}-s_{\beta} e^{i \gamma^{\prime}} U_{e 3}\right|\right), \\
\delta^{\prime \prime}= & -\arg U_{e 3}^{\prime \prime} \arg \left(c_{\beta} e^{-i \gamma^{\prime}} U_{e 1}+s_{\beta} e^{i \gamma^{\prime}} U_{\tau 1}\right)+\arg \left(c_{\beta} e^{-i \gamma^{\prime}} U_{e 2}+s_{\beta} e^{i \gamma^{\prime}} U_{\tau 2}\right), \\
& -\arg \left(c_{\beta} e^{-i \gamma^{\prime}} U_{e 3}+s_{\beta} e^{i \gamma^{\prime}} U_{\tau 3}\right)+\arg \left(c_{\beta} e^{-i \gamma^{\prime}} U_{\tau 3}-s_{\beta} e^{i \gamma^{\prime}} U_{e 3}\right),
\end{aligned}
$$


where $c_{\beta} \equiv \cos \beta, s_{\beta} \equiv \sin \beta$. The inside of the square bracket in the mass matrix (43) has exactly the same form as that of the standard case with replacement $\theta_{j k} \rightarrow \theta_{j k}^{\prime \prime}, \delta \rightarrow \delta^{\prime \prime}$ and $A \rightarrow \lambda_{e^{\prime}}$. Furthermore, at the two level-crossings specified by $\Delta E_{31} \cos 2 \theta_{13}^{\prime \prime}=\lambda_{e^{\prime}}$ and $\Delta E_{21} \cos 2 \theta_{12}^{\prime \prime}=\left(c_{13}^{\prime \prime}\right)^{2} \lambda_{e^{\prime}}, \tilde{\theta}_{13}^{\prime \prime}$ and $\tilde{\theta}_{12}^{\prime \prime}$ become $\pi / 4$, respectively. Therefore, $\tilde{\theta}_{13}^{\prime \prime}$ and $\tilde{\theta}_{12}^{\prime \prime}$ can be regarded as the appropriate mixing angles to describe the nonadiabatic transition at the two level-crossings. Hence we can deduce the jumping factors at the two level-crossings ${ }^{8}$ :

$$
\begin{aligned}
P_{H} & =\exp \left(-\frac{\pi}{2} \cdot \frac{\Delta E_{31} \sin ^{2} 2 \theta_{13}^{\prime \prime}}{\cos 2 \theta_{13}^{\prime \prime}|d \log A / d t|_{\text {resonance }}}\right) \\
P_{L} & =\exp \left(-\frac{\pi}{2} \cdot \frac{\Delta E_{21} \sin ^{2} 2 \theta_{12}^{\prime \prime}}{\cos 2 \theta_{12}^{\prime \prime}|d \log A / d t|_{\text {resonance }}}\right)
\end{aligned}
$$

To estimate the effective mixing matrix elements at the origin $L=0$, we assume that the matter effect $A$ is much larger than the energy difference $\left|\Delta E_{j k}\right|$. In this case we can ignore the term $\mathcal{E}$ in Eq. (53), and Eq. (41) indicates that the mixing matrix $\tilde{U}$ is given by $e^{i \gamma^{\prime} \lambda_{9}} e^{-i \beta \lambda_{5}}$, and we get

$$
\left|\tilde{U}_{\alpha j}(0)\right|^{2}=\left(\begin{array}{ccc}
c_{\beta}^{2} & 0 & s_{\beta}^{2} \\
0 & 1 & 0 \\
s_{\beta}^{2} & 0 & c_{\beta}^{2}
\end{array}\right) .
$$

From Eqs. (11), (48), (49) and (150), we can obtain the transition probability $P\left(\nu_{\alpha} \rightarrow \nu_{\beta}\right)$ in the case with the nonstandard neutrino interaction in propagation.

\section{B. The case with large magnetic moments and a magnetic field}

The second example is the case where there are three active neutrinos with magnetic moments and a large magnetic field ${ }^{9}$. This is an example where the energy eigenvalues cannot be expressed as roots of a quadratic equation, and this case demonstrates the usefulness of the KTY formalism. Here we assume the magnetic interaction of Majorana type

$$
\mu_{\alpha \beta} \bar{\nu}_{\alpha} F_{\lambda \kappa} \sigma^{\lambda \kappa} \nu_{\beta}^{c}+\text { h.c. }
$$

and in this case the magnetic moments $\mu_{\alpha \beta}$ are real and anti-symmetric in flavor indices: $\mu_{\alpha \beta}=-\mu_{\beta \alpha}$. The hermitian matrix ${ }^{10}$

$$
\mathcal{M} \equiv\left(\begin{array}{cc}
U \mathcal{E} U^{-1} & \mathcal{B} \\
\mathcal{B}^{\dagger} & U^{*} \mathcal{E}\left(U^{*}\right)^{-1}
\end{array}\right)
$$

with

$$
\mathcal{B}_{\alpha \beta} \equiv B \mu_{\alpha \beta}
$$

8 The quantity $P_{L}$ was given first in Ref. [22] whose result agrees with ours.

9 The possibility that the magnetic moments of neutrinos in a large magnetic field affect the neutrino flavor transition caught a lot of attention after this idea was applied to the solar neutrino deficit in Refs. 23 26].

10 See Ref. [15] for derivation of Eq. (52) from the Dirac Eq. 
is the mass matrix for neutrinos and anti-neutrinos without the matter effect where neutrinos have the magnetic moments $\mu_{\alpha \beta}$ in the magnetic field $B$.

For simplicity we consider the limit $\theta_{13} \rightarrow 0$ and $\Delta m_{21}^{2} \rightarrow 0$, and we assume that all the $\mathrm{CP}$ phases vanish ${ }^{11}$. Then the matrix (52) can be rewritten as

$$
\mathcal{M}=\frac{1}{2}\left(\begin{array}{rr}
\mathbf{1} & i \mathbf{1} \\
i \mathbf{1} & \mathbf{1}
\end{array}\right)\left(\begin{array}{cc}
U \mathcal{E} U^{-1}+i \mathcal{B} & 0 \\
0 & U \mathcal{E} U^{-1}-i \mathcal{B}
\end{array}\right)\left(\begin{array}{rr}
\mathbf{1} & -i \mathbf{1} \\
-i \mathbf{1} & \mathbf{1}
\end{array}\right)
$$

so the problem of diagonalizing the $6 \times 6$ matrix $(52)$ is reduced to diagonalizing the $3 \times 3$ matrices $U \mathcal{E} U^{-1} \pm i \mathcal{B}$. Since we are assuming that all the $\mathrm{CP}$ phases vanish, all the matrix elements $U_{\alpha j}$ and $\mathcal{B}_{\alpha \beta}=-\mathcal{B}_{\beta \alpha}$ are real, $U \mathcal{E} U^{-1} \pm i \mathcal{B}$ can be diagonalized by a unitary matrix and its complex conjugate:

$$
\begin{aligned}
U \mathcal{E} U^{-1}+i \mathcal{B} & =\tilde{U} \tilde{\mathcal{E}} \tilde{U}^{-1} \\
U \mathcal{E} U^{-1}-i \mathcal{B} & =\tilde{U}^{*} \tilde{\mathcal{E}}\left(\tilde{U}^{*}\right)^{-1}
\end{aligned}
$$

and the equation for motion is given by

$$
\frac{d}{d t}\left(\begin{array}{c}
\Psi(t)+i \Psi^{c}(t) \\
\Psi(t)-i \Psi^{c}(t)
\end{array}\right)=\left(\begin{array}{c}
\tilde{U}(t) \tilde{\mathcal{E}}(t) \tilde{U}^{-1}(t)\left\{\Psi(t)+i \Psi^{c}(t)\right\} \\
\tilde{U}^{*}(t) \tilde{\mathcal{E}}(t)\left(\tilde{U}^{*}\right)^{-1}(t)\left\{\Psi(t)-i \Psi^{c}(t)\right\}
\end{array}\right)
$$

To evaluate the energy eigenvalues and the jumping probability, let us simplify the matrix (53). Introducing the notations

$$
\begin{aligned}
\mathcal{B}_{\alpha \beta} & =B \mu_{\alpha \beta} \equiv\left(\begin{array}{ccc}
0 & -p_{0} & -q_{0} \\
p_{0} & 0 & -r_{0} \\
q_{0} & r_{0} & 0
\end{array}\right), \\
e^{-i \theta_{23} \lambda_{7}} \mathcal{B} e^{i \theta_{23} \lambda_{7}} & =\left(\begin{array}{ccc}
0 & -p_{0} c_{23}+q_{0} s_{23} & -p_{0} s_{23}-q_{0} c_{23} \\
p_{0} c_{23}-q_{0} s_{23} & 0 & -r_{0} \\
p_{0} s_{23}+q_{0} c_{23} & r_{0} & 0
\end{array}\right) \\
& \equiv\left(\begin{array}{ccc}
0 & -p & -q \\
p & 0 & -r \\
q & r & 0
\end{array}\right),
\end{aligned}
$$

it is shown in Appendix D that Eq. (53) can be rewritten as

$$
e^{i \theta_{23} \lambda_{7}} e^{i \omega \lambda_{2}}\left[\operatorname{diag}\left(0,0, \Delta E_{31}\right)+\Lambda e^{i \chi \lambda_{5}} \lambda_{2} e^{-i \chi \lambda_{5}}\right] e^{-i \omega \lambda_{2}} e^{-i \theta_{23} \lambda_{7}}
$$

where $\Lambda, \omega$ and $\chi$ are defined by

$$
\begin{aligned}
& \Lambda \equiv \sqrt{p^{2}+q^{2}+r^{2}}, \\
& \omega \equiv \tan ^{-1} \frac{r}{q} \\
& \chi \equiv \tan ^{-1} \frac{\sqrt{q^{2}+r^{2}}}{p} .
\end{aligned}
$$

11 In the presence of the magnetic interaction (51) of Majorana type, the two CP phases, which are absorbed by redefinition of the charged lepton fields in the standard case, cannot be absorbed and therefore become physical. Here, however, we assume for simplicity that these CP phases vanish. 


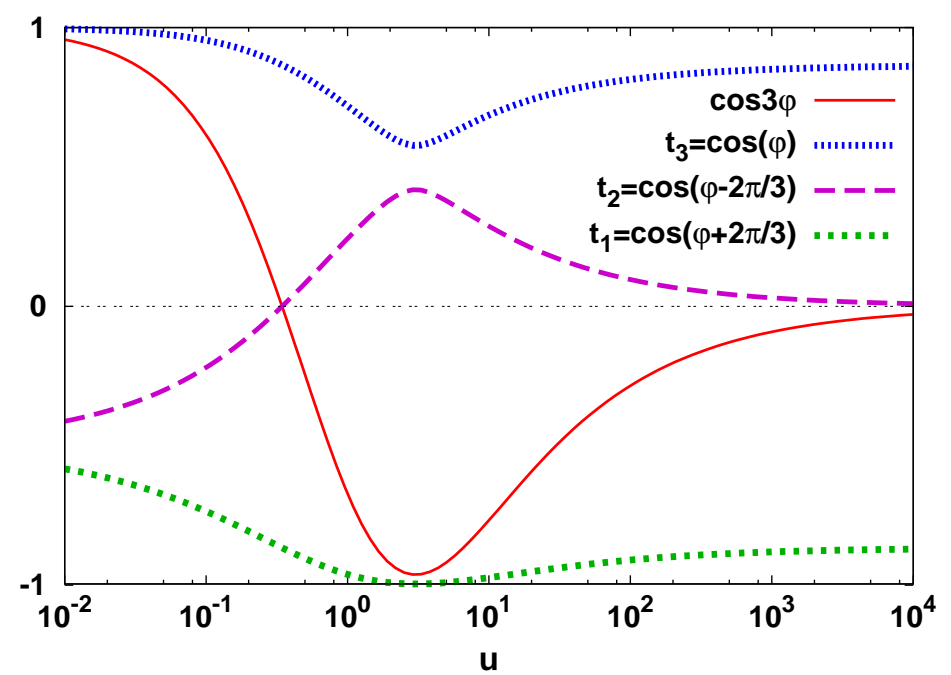

FIG. 1: The behaviors of the normalized eigenvalues $t_{j} \equiv \tilde{E}_{j} / 2 \sqrt{\Delta E_{31}^{2} / 9+\Lambda^{2} / 3}=\cos (\varphi+$ $2 j \pi / 3)(j=1,2,3)$ of $\mathcal{M}$ and $\cos 3 \varphi$ as functions of $u \equiv 3 \Lambda^{2} / \Delta E_{31}^{2}$. See Appendix $\mathrm{E}$ for details.

Since the we are mainly interested in the effective mixing angle which mixes the two energy eigenstates, the matrices $e^{i \theta_{23} \lambda_{7}} e^{i \omega \lambda_{2}}$ on the left-hand side and $e^{-i \omega \lambda_{2}} e^{-i \theta_{23} \lambda_{7}}$ on the right-hand side of the square bracket in Eq. (56) are irrelevant, so we discuss the following matrix:

$$
\mathcal{M} \equiv \operatorname{diag}\left(0,0, \Delta E_{31}\right)-\frac{\Delta E_{31}}{3} \mathbf{1}+\Lambda e^{i \chi \lambda_{5}} \lambda_{2} e^{-i \chi \lambda_{5}},
$$

where a matrix which is proportional to identity was subtracted for convenience in later calculations so that the trace of $\mathcal{M}$ vanishes.

The eigenvalues of the matrix $\mathcal{M}$ are given in Appendix E In Fig. 1 the three eigenvalues $t_{j}(j=1,2,3)$ which are normalized by $2 \sqrt{\Delta E_{31}^{2} / 9+\Lambda^{2} / 3}$ are depicted as a function of $u \equiv 3 \Lambda^{2} / \Delta E_{31}^{2}$. If $\chi$ is small, then the two of the three eigenvalues get close to each other, and $\chi$ can be regarded as the vacuum mixing angle near the level-crossing in the present case. In this example, for a large value of $\Lambda \gg \Delta E_{31}$, the energy eigenvalues are 0 and $\pm \Lambda$, and we found that there is only one level-crossing for $\left|\Delta E_{31}\right| \sim \Lambda$, unlike in the standard three flavor case ${ }^{12}$. So in the following we discuss the contribution from one level-crossing only.

Furthermore, it is shown in Appendix $\mathrm{F}$ that the following relation holds:

$$
P\left(\nu_{\alpha} \rightarrow \nu_{\beta}\right)+P\left(\nu_{\alpha} \rightarrow \bar{\nu}_{\beta}\right)=\sum_{j, k}\left|\tilde{U}_{\beta j}(L)\right|^{2}\left|W_{j k}\right|^{2}\left|\tilde{U}_{\alpha k}^{*}(0)\right|^{2}
$$

12 In principle one could say that the other level-crossing is at $\Lambda=0$, i.e., in vacuum. However, in vacuum the jumping factor $P_{C}$ vanishes, so that the matrix which involves $P_{L}$ in Eq. (11) becomes identity. Hence there is only only one level-crossing in practice. 


$$
=\left(\begin{array}{lll}
\left|U_{\beta 1}\right|^{2} & \left|U_{\beta 2}\right|^{2} & \left|U_{\beta 3}\right|^{2}
\end{array}\right)\left(\begin{array}{ccc}
1 & 0 & 0 \\
0 & 1-P_{H} & P_{H} \\
0 & P_{H} & 1-P_{H}
\end{array}\right)\left(\begin{array}{c}
\left|\tilde{U}_{\alpha 1}(0)\right|^{2} \\
\left|\tilde{U}_{\alpha 2}(0)\right|^{2} \\
\left|\tilde{U}_{\alpha 3}(0)\right|^{2}
\end{array}\right) \text {, }
$$

where we have assumed that the level-crossing occurs between the energy eigenstates 2 and 3 , and we have assumed that there is no magnetic field at the endpoint $t=L$.

As is explained in detail in Appendix G, in the approximation of the small mixing angle $\chi$, the jumping factor $P_{H}$ can be calculated as

$$
P_{H} \simeq \exp \left(-\frac{\pi}{|d \Lambda / d t|_{u=u_{0}}} \Delta E_{31}^{2} \chi^{2}\right)
$$

Let us now check whether the exponent of $P_{H}$ is proportional to the factor $\gamma$. For this purpose, we introduce the parametrization of the mixing matrix other than the standard one:

$$
\begin{aligned}
U & =e^{i \psi_{12} \lambda_{2}} \Gamma_{\eta}^{(13)} e^{i \psi_{13} \lambda_{5}}\left(\Gamma_{\eta}^{(13)}\right)^{-1} e^{i \psi_{23} \lambda_{7}} \\
& =\left(\begin{array}{ccc}
s_{12} c_{13} & s_{12} c_{23}-e^{-i \eta} c_{12} s_{13} s_{23} & s_{12} s_{23}+e^{-i \eta} c_{12} s_{13} c_{23} \\
-s_{12} c_{13} & c_{12} c_{23}+e^{-i \eta} s_{12} s_{13} s_{23} & c_{12} s_{23}-e^{-i \eta} s_{12} s_{13} c_{23} \\
-e^{i \eta} s_{13} & -c_{13} s_{23} & c_{13} c_{23}
\end{array}\right),
\end{aligned}
$$

where $c_{j k} \equiv \cos \psi_{j k}, s_{j k} \equiv \sin \psi_{j k}$, and we have used the notations $\psi_{j k}$ and $\eta$ which are different from those of the standard parametrization (B1) to avoid confusion. From this expression we observe

$$
\tan ^{2} \tilde{\psi}_{23}=\frac{\tilde{X}_{2}^{\tau \tau}}{\tilde{X}_{3}^{\tau \tau}}
$$

where $\tilde{X}_{j}^{\tau \tau}(j=2,3)$ can be obtained from Eq. (17) by replacing $U \mathcal{E} U^{-1}+\mathcal{A} \rightarrow \mathcal{M}$. As is shown in Appendix $\left[\right.$, the effective mixing angle $\tilde{\psi}_{23}$ becomes maximal at the level-crossing. So $\tilde{\psi}_{23}$ is the appropriate mixing angle to describe the nonadiabatic transition between the two energy eigenstates with $\tilde{E}_{2}$ and $\tilde{E}_{3}$.

In Appendix $\left[\mathrm{H}\right.$ it is also shown that the exponent of the jumping factor $P_{H}$ coincides with $-\pi / 2$ times the $\gamma$ factor in the case of a linear potential $(F=1)$ :

$$
\gamma=\left.\frac{\Delta \tilde{E}_{32}}{2\left|d \tilde{\psi}_{23} / d t\right|_{u=u_{0}}}\right|_{u=u_{0}} \simeq \frac{2 \Delta E_{31}^{2} \chi^{2}}{|d \Lambda / d t|_{u=u_{0}}}=-\frac{\log P_{H}}{\pi / 2} .
$$

To obtain the transition probability we also need the expression for $\left|\tilde{U}_{\alpha j}(0)\right|^{2}$. We can roughly estimate the elements $\left|\tilde{U}_{\alpha j}(0)\right|^{2}$ of the effective mixing matrix at the origin $t=0$ by ignoring the term $\mathcal{E}$ in Eq. (53). Using the property (D2) we see

$$
\begin{aligned}
\mathcal{B} & =\left(\begin{array}{ccc}
0 & -p_{0} & -q_{0} \\
p_{0} & 0 & -r_{0} \\
q_{0} & r_{0} & 0
\end{array}\right)=\Lambda e^{i \omega_{0} \lambda_{2}} e^{i \chi_{0} \lambda_{5}} \lambda_{2} e^{-i \chi_{0} \lambda_{5}} e^{-i \omega_{0} \lambda_{2}} \\
& =\Lambda e^{i \omega_{0} \lambda_{2}} e^{i \chi_{0} \lambda_{5}} e^{i(\pi / 4) \lambda_{1}} \lambda_{3} e^{-i(\pi / 4) \lambda_{1}} e^{-i \chi_{0} \lambda_{5}} e^{-i \omega_{0} \lambda_{2}},
\end{aligned}
$$


where the angles $\omega_{0}$ and $\chi_{0}$ are defined by $\omega_{0} \equiv \tan ^{-1}\left(r_{0} / q_{0}\right), \chi_{0} \equiv \tan ^{-1}\left(\sqrt{q_{0}^{2}+r_{0}^{2}} / p_{0}\right)$. Eq. (63) implies that the effective mixing matrix $\tilde{U}$ at the origin is $\tilde{U}=e^{i \omega_{0} \lambda_{2}} e^{i \chi_{0} \lambda_{5}} e^{i(\pi / 4) \lambda_{1}}$, so that we have

$$
\begin{aligned}
\left|\tilde{U}_{\alpha j}(0)\right|^{2} & =\left(\begin{array}{ccc}
\left|\frac{1}{\sqrt{2}}\left(c_{\omega_{0}} c_{\chi_{0}}+i s_{\omega_{0}}\right)\right|^{2} & \left|\frac{1}{\sqrt{2}}\left(i c_{\omega_{0}} c_{\chi_{0}}+s_{\omega_{0}}\right)\right|^{2} & \left|c_{\omega_{0}} s_{\chi_{0}}\right|^{2} \\
\left|\frac{1}{\sqrt{2}}\left(-s_{\omega_{0}} c_{\chi_{0}}+i c_{\omega_{0}}\right)\right|^{2} & \left|\frac{1}{\sqrt{2}}\left(-i s_{\omega_{0}} c_{\chi_{0}}+c_{\omega_{0}}\right)\right|^{2} & \left|-s_{\omega_{0}} s_{\chi_{0}}\right|^{2} \\
\left|-\frac{1}{\sqrt{2}} s_{\chi_{0}}\right|^{2} & \left|-\frac{i}{\sqrt{2}} s_{\chi_{0}}\right|^{2} & \left|c_{\chi_{0}}\right|^{2}
\end{array}\right) \\
& =\frac{1}{2 \Lambda^{2}\left(q_{0}^{2}+r_{0}^{2}\right)}\left(\begin{array}{ccc}
r_{0}^{2} \Lambda^{2}+p_{0}^{2} q_{0}^{2} & r_{0}^{2} \Lambda^{2}+p_{0}^{2} q_{0}^{2} & 2 q_{0}^{2}\left(q_{0}^{2}+r_{0}^{2}\right) \\
q_{0}^{2} \Lambda^{2}+p_{0}^{2} r_{0}^{2} & q_{0}^{2} \Lambda^{2}+p_{0}^{2} r_{0}^{2} & 2 r_{0}^{2}\left(q_{0}^{2}+r_{0}^{2}\right) \\
\left(q_{0}^{2}+r_{0}^{2}\right)^{2} & \left(q_{0}^{2}+r_{0}^{2}\right)^{2} & 2 p_{0}^{2}\left(q_{0}^{2}+r_{0}^{2}\right)
\end{array}\right) .
\end{aligned}
$$

From Eqs. (61), (62) and (64), we obtain the combination $P\left(\nu_{\alpha} \rightarrow \nu_{\beta}\right)+P\left(\nu_{\alpha} \rightarrow \bar{\nu}_{\beta}\right)$ of the transition probabilities.

In the discussions above we have assumed that $|\chi|$ is small. If this is not the case, then $\tilde{\psi}_{23}$ does not necessarily become maximal at the level-crossing. In that case, instead of the matrix $\mathcal{M}$ in Eq. (60), we should use

$$
\mathcal{M}^{\prime} \equiv e^{-i \alpha \lambda_{5}} \mathcal{M} e^{i \alpha \lambda_{5}} \equiv e^{-i \alpha \lambda_{5}} \tilde{U} \tilde{\mathcal{E}} \tilde{U}^{-1} e^{i \alpha \lambda_{5}} \equiv \tilde{U}^{\prime} \tilde{\mathcal{E}}\left(\tilde{U}^{\prime}\right)^{-1}
$$

$\alpha$ is a parameter which is determined by the condition

$$
\left.\tan ^{2} \tilde{\psi}_{23}^{\prime}\right|_{u=u_{0}}=\left.\frac{\tilde{X}_{2}^{\prime \tau \tau}}{\tilde{X}_{3}^{\prime \tau \tau}}\right|_{u=u_{0}}=\left.\frac{\left|\tilde{U}_{\tau 2} \cos \alpha+\tilde{U}_{e 2} \sin \alpha\right|^{2}}{\left|\tilde{U}_{\tau 3} \cos \alpha+\tilde{U}_{e 3} \sin \alpha\right|^{2}}\right|_{u=u_{0}}=1
$$

The condition (66) is a quadratic equation with respect to $\tan \alpha$ :

$$
\left(\left|\tilde{U}_{e 2}\right|^{2}-\left|\tilde{U}_{e 3}\right|^{2}\right) \tan ^{2} \alpha+2 \operatorname{Re}\left(\tilde{U}_{e 2} \tilde{U}_{\tau 2}^{*}-\tilde{U}_{e 3} \tilde{U}_{\tau 3}^{*}\right) \tan \alpha+\left|\tilde{U}_{\tau 2}\right|^{2}-\left|\tilde{U}_{\tau 3}\right|^{2}=0
$$

and the discriminant of Eq. (67) is given by

$$
\left\{\operatorname{Re}\left(\tilde{U}_{e 2} \tilde{U}_{\tau 2}^{*}-\tilde{U}_{e 3} \tilde{U}_{\tau 3}^{*}\right)\right\}^{2}-\left(\left|\tilde{U}_{e 2}\right|^{2}-\left|\tilde{U}_{e 3}\right|^{2}\right)\left(\left|\tilde{U}_{\tau 2}\right|^{2}-\left|\tilde{U}_{\tau 3}\right|^{2}\right) .
$$

In the present case, the quantity $\tilde{X}_{j}^{e \tau} \equiv \tilde{U}_{e j} \tilde{U}_{\tau j}^{*}(j=2,3)$ turns out to be real because $\left(\mathcal{M}^{j-1}\right)_{e \tau}(j=2,3)$ is real. Hence the discriminant (68) becomes $\left(\tilde{U}_{e 2} \tilde{U}_{\tau 3}-\tilde{U}_{e 3} \tilde{U}_{\tau 2}\right)^{2}$ which is positive semi-definite, and the quadratic equation (67) always has real roots. Using one of the roots of (67) as tan $\alpha$ in Eq. (65), we can evaluate the $\gamma$ factor. Thus, on the assumption that the jumping probability $P_{H}$ is given by the factor $\exp (-\gamma F \pi / 2)$, we can deduce the jumping factor $P_{H}$. In this case, however, unlike in the case of $|\chi| \ll 1$, we cannot prove that the exponent of the jumping probability (9) in the WKB treatment is equal to $-\gamma F \pi / 2$.

\section{CONCLUSIONS}

Using the formalism which was developed by Kimura, Takamura and Yokomakura to express analytically the combination $\tilde{X}_{j}^{\alpha \beta} \equiv \tilde{U}_{\alpha j} \tilde{U}_{\beta j *}$ of the mixing matrix elements in matter with constant density, we have shown that the effective mixing angle can be analytically expressed in terms of the mixing matrix elements in vacuum and the energy eigenvalues. The analytical expression for the effective mixing angle enables us to evaluate the nonadiabatic 
contribution to the transition probability based on the two assumptions: (i) The nonadiabatic transitions in the case with more than two energy eigenstates can be separately treated as a two state problem at each level-crossing. (ii) The exponent of the probability obtained by the WKB method is proportional to the factor $\gamma$ which is the ratio of the energy difference of the two eigenstates to the derivative of the effective mixing angle at the level-crossing. We have given two examples: one with flavor dependent nonstandard interactions in neutrino propagation and the other with magnetic moments in a large magnetic field. In the second example the energy eigenvalues cannot be expressed as roots of a quadratic equation and discussions become much less trivial compared with the standard case or with the first example. In the second example we have shown in the approximation of the small mixing angle that the above assumption (ii) is correct. If the two assumptions (i) and (ii) above are correct, then the KTY formalism enables us to express the probability of nonadiabatic transitions in terms of the mixing matrix elements in vacuum and the energy eigenvalues in general cases.

\section{Appendix A: The effective mixing angle in the standard three flavor case}

Near the level-crossing $\Delta E_{31} \simeq A$, in the leading order in $\Delta E_{21} / \Delta E_{31}$, the mass matrix (2) becomes

$$
\begin{aligned}
& U \mathcal{E} U^{-1}+\mathcal{A} \\
= & e^{i \theta_{23} \lambda_{7}} \Gamma_{\delta}^{(13)} e^{i \tilde{\theta}_{13} \lambda_{5}} \frac{1}{2} \operatorname{diag}\left(\Delta E_{31}+A-\Delta \tilde{E}_{31}, 0, \Delta E_{31}+A+\Delta \tilde{E}_{31}\right) \\
& \times e^{-i \tilde{\theta}_{13} \lambda_{5}}\left(\Gamma_{\delta}^{(13)}\right)^{-1} e^{-i \theta_{23} \lambda_{7}},
\end{aligned}
$$

where $\lambda_{j}(j=5,7)$ are defined by Eqs. (33) and (34), and

$$
\begin{aligned}
\tan 2 \tilde{\theta}_{13} & \equiv \frac{\Delta E_{31} \sin 2 \theta_{13}}{\Delta E_{31} \cos 2 \theta_{13}-A} \\
\Delta \tilde{E}_{31} & \equiv \sqrt{\left(\Delta E_{31} \cos 2 \theta_{13}-A\right)^{2}+\left(\Delta E_{31} \sin 2 \theta_{13}\right)^{2}} \\
\Gamma_{\delta}^{(13)} & \equiv \operatorname{diag}\left(e^{-i \delta / 2}, 1, e^{i \delta / 2}\right)
\end{aligned}
$$

At $\Delta E_{31} \cos 2 \theta_{13}=A$, the effective mixing angle $\tilde{\theta}_{13}$ becomes $\pi / 4$, as in the two flavor case. Hence $\tilde{\theta}_{13}$ can be regarded as the appropriate mixing angle to describe the nonadiabatic transition between the two energy eigenstates with $\tilde{E}_{1}$ and $\tilde{E}_{3}$.

On the other hand, near the level-crossing $\Delta E_{21} \simeq A$, to first order in $\Delta E_{21} / \Delta E_{31}$ the mass matrix (2) becomes

$$
\begin{aligned}
& U \mathcal{E} U^{-1}+\mathcal{A} \\
= & e^{i \theta_{23} \lambda_{7}} \Gamma_{\delta}^{(13)} e^{i \theta_{13} \lambda_{5}}\left(\Gamma_{\delta}^{(13)}\right)^{-1} \exp \left(i \sum_{j=4}^{7} d_{j} \lambda_{j}\right) e^{i \tilde{\theta}_{12} \lambda_{2}} \\
& \times\left[\operatorname{diag}\left\{\frac{1}{2}\left(\Delta E_{21}+A c_{13}^{2}+\Delta \tilde{E}_{21}, \Delta E_{21}+A c_{13}^{2}-\Delta \tilde{E}_{21}, \Delta E_{31}+A s_{13}^{2}\right\}\right]\right. \\
& \times e^{-i \tilde{\theta}_{12} \lambda_{2}} \exp \left(-i \sum_{j=4}^{7} d_{j} \lambda_{j}\right) \Gamma_{\delta}^{(13)} e^{-i \theta_{13} \lambda_{5}}\left(\Gamma_{\delta}^{(13)}\right)^{-1} e^{-i \theta_{23} \lambda_{7}},
\end{aligned}
$$


where $\lambda_{2}$ is defined by Eq. (32),

$$
\begin{aligned}
\tan 2 \tilde{\theta}_{12} & \equiv \frac{\Delta E_{21} \sin 2 \theta_{12}}{\Delta E_{21} \cos 2 \theta_{12}-A c_{13}^{2}} \\
\Delta \tilde{E}_{21} & \equiv \sqrt{\left(\Delta E_{21} \cos 2 \theta_{12}-A c_{13}^{2}\right)^{2}+\left(\Delta E_{21} \sin 2 \theta_{12}\right)^{2}}
\end{aligned}
$$

and $d_{j}(j=4, \cdots, 7)$ are the small coefficients given by $d_{4}=c_{12} \sin \delta A / \Delta E_{31}, d_{5}=$ $c_{12} \cos \delta A / \Delta E_{31}, d_{6}=s_{12} \sin \delta A / \Delta E_{31}$ and $d_{7}=s_{12} \cos \delta A / \Delta E_{31}$. At $\Delta E_{21} \cos 2 \theta_{12}=A c_{13}^{2}$, the effective mixing angle $\tilde{\theta}_{12}$ becomes $\pi / 4$. Hence $\tilde{\theta}_{12}$ can be regarded as the appropriate

mixing angle to describe the nonadiabatic transition between the two energy eigenstates with $\widetilde{E}_{1}$ and $\tilde{E}_{2}$.

\section{Appendix B: Parametrizations for $3 \times 3$ unitary matrices}

Assuming the same form as for the standard parametrization, we can consider six permutations for $3 \times 3$ unitary matrices as follows:

$$
\begin{aligned}
& U=e^{i \theta_{23} \lambda_{7}} \Gamma_{\delta}^{(13)} e^{i \theta_{13} \lambda_{5}}\left(\Gamma_{\delta}^{(13)}\right)^{-1} e^{i \theta_{12} \lambda_{2}} \\
& =\left(\begin{array}{ccc}
1 & 0 & 0 \\
0 & c_{23} & s_{23} \\
0 & -s_{23} & c_{23}
\end{array}\right)\left(\begin{array}{ccc}
e^{-i \delta / 2} & 0 & 0 \\
0 & 1 & 0 \\
0 & 0 & e^{i \delta / 2}
\end{array}\right)\left(\begin{array}{ccc}
c_{13} & 0 & s_{13} \\
0 & 1 & 0 \\
-s_{13} & 0 & c_{13}
\end{array}\right) \\
& \times\left(\begin{array}{ccc}
e^{i \delta / 2} & 0 & 0 \\
0 & 1 & 0 \\
0 & 0 & e^{-i \delta / 2}
\end{array}\right)\left(\begin{array}{ccc}
c_{12} & s_{12} & 0 \\
-s_{12} & c_{12} & 0 \\
0 & 0 & 1
\end{array}\right), \\
& =\left(\begin{array}{ccc}
c_{12} c_{13} & s_{12} c_{13} & s_{13} e^{-i \delta} \\
-s_{12} c_{23}-c_{12} s_{23} s_{13} e^{i \delta} & c_{12} c_{23}-s_{12} s_{23} s_{13} e^{i \delta} & s_{23} c_{13} \\
s_{12} s_{23}-c_{12} c_{23} s_{13} e^{i \delta} & -c_{12} s_{23}-s_{12} c_{23} s_{13} e^{i \delta} & c_{23} c_{13}
\end{array}\right) \text {, } \\
& U=e^{i \theta_{13} \lambda_{5}} \Gamma_{\delta}^{(23)} e^{i \theta_{23} \lambda_{7}}\left(\Gamma_{\delta}^{(23)}\right)^{-1} e^{i \theta_{12} \lambda_{2}} \\
& =\left(\begin{array}{ccc}
c_{12} c_{13}+e^{i \delta} s_{12} s_{13} s_{23} & s_{12} c_{13}-e^{i \delta} c_{12} s_{13} s_{23} & s_{13} c_{23} \\
-s_{12} c_{23} & c_{12} c_{23} & s_{23} e^{-i \delta} \\
c_{12} s_{13}+e^{i \delta} s_{12} c_{13} s_{23} & -s_{12} s_{13}-e^{i \delta} c_{12} c_{13} s_{23} & c_{13} c_{23}
\end{array}\right), \\
& U=e^{i \theta_{23} \lambda_{7}} \Gamma_{\delta}^{(12)} e^{i \theta_{12} \lambda_{2}}\left(\Gamma_{\delta}^{(12)}\right)^{-1} e^{i \theta_{13} \lambda_{5}} \\
& =\left(\begin{array}{ccc}
c_{12} c_{13} & e^{-i \delta} s_{12} & c_{12} s_{13} \\
-s_{13} s_{23}-e^{i \delta} s_{12} c_{13} c_{23} & c_{12} c_{23} & c_{13} s_{23}-e^{i \delta} s_{12} s_{13} c_{23} \\
-s_{13} c_{23}-e^{i \delta} s_{12} c_{13} s_{23} & -c_{12} s_{23} & c_{13} c_{23}+e^{i \delta} s_{12} s_{13} s_{23}
\end{array}\right), \\
& U=e^{i \theta_{12} \lambda_{2}} \Gamma_{\delta}^{(23)} e^{i \theta_{23} \lambda_{7}}\left(\Gamma_{\delta}^{(23)}\right)^{-1} e^{i \theta_{13} \lambda_{5}} \\
& =\left(\begin{array}{ccc}
c_{12} c_{13}-e^{-i \delta} s_{12} s_{13} s_{23} & s_{12} c_{23} & c_{12} s_{13}-e^{-i \delta} s_{12} c_{13} s_{23} \\
-s_{12} c_{13}-e^{-i \delta} c_{12} s_{13} s_{23} & c_{12} c_{23} & -s_{12} s_{13}+e^{-i \delta} c_{12} c_{13} s_{23} \\
-s_{13} c_{23} & -e^{i \delta} s_{23} & c_{13} c_{23}
\end{array}\right),
\end{aligned}
$$




$$
\begin{aligned}
U & =e^{i \theta_{12} \lambda_{2}} \Gamma_{\delta}^{(13)} e^{i \theta_{13} \lambda_{5}}\left(\Gamma_{\delta}^{(13)}\right)^{-1} e^{i \theta_{23} \lambda_{7}} \\
& =\left(\begin{array}{ccc}
c_{12} c_{13} & s_{12} c_{23}-e^{-i \delta} c_{12} s_{13} s_{23} & s_{12} s_{23}+e^{-i \delta} c_{12} s_{13} s_{23} \\
-s_{12} c_{13} & c_{12} c_{23}+e^{-i \delta} s_{12} s_{13} s_{23} & c_{12} s_{23}-e^{-i \delta} s_{12} s_{13} c_{23} \\
-e^{i \delta} s_{13} & -c_{13} s_{23} & c_{13} c_{23}
\end{array}\right), \\
U & =e^{i \theta_{13} \lambda_{2}} \Gamma_{\delta}^{(12)} e^{i \theta_{12} \lambda_{5}}\left(\Gamma_{\delta}^{(12)}\right)^{-1} e^{i \theta_{23} \lambda_{7}} \\
& =\left(\begin{array}{ccc}
c_{12} c_{13} & -s_{13} c_{23}+e^{-i \delta} s_{12} c_{13} c_{23} & s_{13} c_{23}+e^{-i \delta} s_{12} c_{13} s_{23} \\
-e^{i \delta} s_{12} & c_{12} c_{23} & c_{12} s_{23} \\
-c_{12} s_{13} & -c_{13} s_{23}-e^{-i \delta} s_{12} s_{13} c_{23} & c_{13} c_{23}-e^{-i \delta} s_{12} s_{13} s_{23}
\end{array}\right) .
\end{aligned}
$$

Here we have introduced the notation:

$$
\begin{aligned}
\Gamma_{\delta}^{(12)} & \equiv \operatorname{diag}\left(e^{-i \delta / 2}, e^{i \delta / 2}, 1\right), \\
\Gamma_{\delta}^{(13)} & \equiv \operatorname{diag}\left(e^{-i \delta / 2}, 1, e^{i \delta / 2}\right), \\
\Gamma_{\delta}^{(23)} & \equiv \operatorname{diag}\left(1, e^{-i \delta / 2}, e^{i \delta / 2}\right) .
\end{aligned}
$$

\section{Appendix C: The derivation of Eq. (43)}

Because of the form of the matrix $\mathcal{A}_{N P}$ (41), the mass matrix (39) can be written as [5]

$$
\begin{aligned}
& U \mathcal{E} U^{-1}+\mathcal{A}_{N P} \\
= & e^{i \gamma^{\prime} \lambda_{9}} e^{-i \beta \lambda_{5}}\left[e^{i \beta \lambda_{5}} e^{-i \gamma^{\prime} \lambda_{9}} U \mathcal{E} U^{-1} e^{i \gamma^{\prime} \lambda_{9}} e^{-i \beta \lambda_{5}}+\operatorname{diag}\left(\lambda_{e^{\prime}}, 0,0\right)\right] e^{i \beta \lambda_{5}} e^{-i \gamma^{\prime} \lambda_{9}} .
\end{aligned}
$$

Here we introduce the following two unitary matrices:

$$
\begin{aligned}
U^{\prime} & \equiv e^{i \beta \lambda_{5}} e^{-i \gamma^{\prime} \lambda_{9}} U \\
& =\left(\begin{array}{ccc}
c_{\beta} e^{-i \gamma^{\prime}} U_{e 1}+s_{\beta} e^{i \gamma^{\prime}} U_{\tau 1} & c_{\beta} e^{-i \gamma^{\prime}} U_{e 2}+s_{\beta} e^{i \gamma^{\prime}} U_{\tau 2} & c_{\beta} e^{-i \gamma^{\prime}} U_{e 3}+s_{\beta} e^{i \gamma^{\prime}} U_{\tau 3} \\
U_{\mu 1} & U_{\mu 2} & U_{\mu 3} \\
c_{\beta} e^{-i \gamma^{\prime}} U_{\tau 1}-s_{\beta} e^{i \gamma^{\prime}} U_{e 1} & c_{\beta} e^{-i \gamma^{\prime}} U_{\tau 2}-s_{\beta} e^{i \gamma^{\prime}} U_{e 2} & c_{\beta} e^{-i \gamma^{\prime}} U_{\tau 3}-s_{\beta} e^{i \gamma^{\prime}} U_{e 3}
\end{array}\right), \\
U^{\prime \prime} & \equiv e^{i \phi_{3} \lambda_{3}} e^{i \phi_{9} \lambda_{9}} U^{\prime} e^{i \omega_{9} \lambda_{9}} e^{i \omega_{3} \lambda_{3}},
\end{aligned}
$$

where $c_{\beta} \equiv \cos \beta, s_{\beta} \equiv \sin \beta$, and $U^{\prime \prime}$ is in the standard parametrization (B1). The phases $\phi_{3}, \phi_{9}, \omega_{3}$ and $\omega_{9}$ are defined in such a way that the elements $U_{e 1}^{\prime \prime}, U_{e 2}^{\prime \prime}, U_{\mu 3}^{\prime \prime}, U_{\tau 3}^{\prime \prime}$ become real to be consistent with the standard parametrization (B1), and are given by

$$
\begin{aligned}
\phi_{3} & =-\frac{1}{3} \arg U_{e 1}^{\prime}-\frac{1}{3} \arg U_{e 2}^{\prime}-\frac{2}{3} \arg U_{\tau 3}^{\prime}, \\
\phi_{9} & =-\frac{1}{3} \arg U_{e 1}^{\prime}-\frac{1}{3} \arg U_{e 2}^{\prime}+\frac{1}{3} \arg U_{\tau 3}^{\prime}, \\
\omega_{3} & =-\frac{2}{3} \arg U_{e 1}^{\prime}+\frac{1}{3} \arg U_{e 2}^{\prime}-\frac{2}{3} \arg U_{\tau 3}^{\prime}, \\
\omega_{9} & =\frac{1}{3} \arg U_{e 1}^{\prime}+\frac{1}{3} \arg U_{e 2}^{\prime}+\frac{2}{3} \arg U_{\tau 3}^{\prime} .
\end{aligned}
$$


In these expressions, $\arg U_{\alpha j}^{\prime}$ can be read off from Eq. (C22). Thus we obtain the expression for the three mixing angles $\theta_{j k}^{\prime \prime}$ (44), (45), (46) and the Dirac phase $\delta^{\prime \prime}$ (47) in $U^{\prime \prime} 13$ :

$$
\begin{aligned}
\theta_{12}^{\prime \prime}= & \tan ^{-1}\left(U_{e 2}^{\prime \prime} / U_{e 1}^{\prime \prime}\right)=\tan ^{-1}\left(\left|c_{\beta} e^{-i \gamma^{\prime}} U_{e 2}+s_{\beta} e^{i \gamma^{\prime}} U_{\tau 2}\right| /\left|c_{\beta} e^{-i \gamma^{\prime}} U_{e 1}+s_{\beta} e^{i \gamma^{\prime}} U_{\tau 1}\right|\right) \\
\theta_{13}^{\prime \prime}= & \sin ^{-1}\left|U_{e 3}^{\prime \prime}\right|=\sin ^{-1}\left|c_{\beta} e^{-i \gamma^{\prime}} U_{e 3}+s_{\beta} e^{i \gamma^{\prime}} U_{\tau 3}\right| \\
\theta_{23}^{\prime \prime}= & \tan ^{-1}\left(U_{\mu 3}^{\prime \prime} / U_{\tau 3}^{\prime \prime}\right)=\tan ^{-1}\left(U_{\mu 3} /\left|c_{\beta} e^{-i \gamma^{\prime}} U_{\tau 3}-s_{\beta} e^{i \gamma^{\prime}} U_{e 3}\right|\right) \\
\delta^{\prime \prime}= & -\arg U_{e 3}^{\prime \prime} \\
= & \arg \left(c_{\beta} e^{-i \gamma^{\prime}} U_{e 1}+s_{\beta} e^{i \gamma^{\prime}} U_{\tau 1}\right)+\arg \left(c_{\beta} e^{-i \gamma^{\prime}} U_{e 2}+s_{\beta} e^{i \gamma^{\prime}} U_{\tau 2}\right) \\
& -\arg \left(c_{\beta} e^{-i \gamma^{\prime}} U_{e 3}+s_{\beta} e^{i \gamma^{\prime}} U_{\tau 3}\right)+\arg \left(c_{\beta} e^{-i \gamma^{\prime}} U_{\tau 3}-s_{\beta} e^{i \gamma^{\prime}} U_{e 3}\right) .
\end{aligned}
$$

\section{Appendix D: The derivation of Eq. (56)}

Because of the form of the matrix (55), we have

$$
\begin{aligned}
U \mathcal{E} U^{-1}+i \mathcal{B} & =e^{i \theta_{23} \lambda_{7}} \operatorname{diag}\left(0,0, \Delta E_{31}\right) e^{-i \theta_{23} \lambda_{7}}+i\left(\begin{array}{ccc}
0 & -p_{0} & -q_{0} \\
p_{0} & 0 & -r_{0} \\
q_{0} & r_{0} & 0
\end{array}\right) \\
& =e^{i \theta_{23} \lambda_{7}}\left[\operatorname{diag}\left(0,0, \Delta E_{31}\right)+i\left(\begin{array}{ccc}
0 & -p & -q \\
p & 0 & -r \\
q & r & 0
\end{array}\right)\right] e^{-i \theta_{23} \lambda_{7}}
\end{aligned}
$$

$T_{1}=i \lambda_{7}, T_{2}=-i \lambda_{5}, T_{3}=i \lambda_{2}$ are the generators of the $S O(3)$ group, and the real antisymmetric matrix $e^{i \theta_{23} \lambda_{7}} \mathcal{B} e^{-i \theta_{23} \lambda_{7}}=-i\left(p \lambda_{2}+q \lambda_{5}+r \lambda_{7}\right)$ can be rewritten as

$$
\begin{aligned}
-i\left(p \lambda_{2}+q \lambda_{5}+r \lambda_{7}\right) & =-i e^{i \omega \lambda_{2}}\left(p \lambda_{2}+\sqrt{q^{2}+r^{2}} \lambda_{7}\right) e^{-i \omega \lambda_{2}} \\
& =-i e^{i \omega \lambda_{2}} e^{i \chi \lambda_{5}} \Lambda \lambda_{2} e^{-i \chi \lambda_{5}} e^{-i \omega \lambda_{2}}
\end{aligned}
$$

where $\Lambda, \omega$ and $\chi$ are defined in Eqs. (57), (158) and (59). Hence the matrix (D1) becomes

$$
\begin{aligned}
U \mathcal{E} U^{-1}+i \mathcal{B} & =e^{i \theta_{23} \lambda_{7}}\left[\operatorname{diag}\left(0,0, \Delta E_{31}\right)+\Lambda e^{i \omega \lambda_{2}} e^{i \chi \lambda_{5}} \lambda_{2} e^{-i \chi \lambda_{5}} e^{-i \omega \lambda_{2}}\right] e^{-i \theta_{23} \lambda_{7}} \\
& =e^{i \theta_{23} \lambda_{7}} e^{i \omega \lambda_{2}}\left[\operatorname{diag}\left(0,0, \Delta E_{31}\right)+\Lambda e^{i \chi \lambda_{5}} \lambda_{2} e^{-i \chi \lambda_{5}}\right] e^{-i \omega \lambda_{2}} e^{-i \theta_{23} \lambda_{7}}
\end{aligned}
$$

Appendix E: The energy eigenvalues in the case with large magnetic moments and a magnetic field

The eigenvalue of the matrix in Eq. (60) can be obtained from the eigenvalue equation

$$
0=|\tilde{E} \mathbf{1}-\mathcal{M}|=\tilde{E}^{3}-\left(\Lambda^{2}+\frac{\Delta E_{31}^{2}}{3}\right) \tilde{E}-\frac{2}{27} \Delta E_{31}^{3}+\frac{1+3 \cos 2 \chi}{6} \Lambda^{2} \Delta E_{31} .
$$

13 There was an error in the discussion on the phases in Appendix C in Ref. [5] in the case of the non-standard interaction. The expressions of the phases here correct those in Ref. [5]. 
The three roots of the cubic equation (E1) are given by

$$
\begin{aligned}
& \tilde{E}_{1}=2 R \cos \left(\varphi+\frac{2}{3} \pi\right), \\
& \tilde{E}_{2}=2 R \cos \left(\varphi-\frac{2}{3} \pi\right), \\
& \tilde{E}_{3}=2 R \cos \varphi,
\end{aligned}
$$

where

$$
\begin{aligned}
R & \equiv\left(\frac{\Delta E_{31}^{2}}{9}+\frac{\Lambda^{2}}{3}\right)^{1 / 2} \\
\cos 3 \varphi & \equiv \frac{1}{R^{3}}\left\{\left(\frac{\Delta E_{31}}{3}\right)^{3}-\frac{1+3 \cos 2 \chi}{12} \Lambda^{2} \Delta E_{31}\right\}=\frac{1-D u}{(1+u)^{3 / 2}} \\
u & \equiv \frac{3 \Lambda^{2}}{\Delta E_{31}} \\
D & \equiv \frac{3}{4}(1+3 \cos 2 \chi)
\end{aligned}
$$

The extremum of $\cos 3 \varphi$ is given by the condition

$$
0=\frac{d}{d u} \cos 3 \varphi=\frac{d}{d u}\left\{\frac{1-D u}{(1+u)^{3 / 2}}\right\}=\frac{D(u-2-3 / D)}{2(1+u)^{5 / 2}},
$$

SO

$$
u=u_{0} \equiv 2+\frac{3}{D}
$$

gives the condition for the level-crossing. At $u=u_{0}$, the value of $\cos 3 \varphi$ is

$$
\left.\cos 3 \varphi\right|_{u=u_{0}}=\left.\frac{1-D u}{(1+u)^{3 / 2}}\right|_{u=u_{0}}=-\left(\frac{D}{3}\right)^{\frac{3}{2}} \sqrt{\frac{4}{1+D}}
$$

When $|\chi|$ is small, we have

$$
\begin{aligned}
& D \simeq 3-\frac{9}{2} \chi^{2}, \\
& u_{0} \simeq 3+\frac{3}{2} \chi^{2},
\end{aligned}
$$

so that $\left.\cos 3 \varphi\right|_{u=u_{0}}$ approaches -1 :

$$
\left.\cos 3 \varphi\right|_{u=u_{0}} \simeq-1+\frac{27}{16} \chi^{2}
$$

This implies that $\left.\varphi\right|_{u=u_{0}}$ is close to $\pi / 3$ :

$$
\left.\varphi\right|_{u=u_{0}} \simeq \frac{\pi}{3}-\sqrt{\frac{3}{8}} \chi
$$

At $u=0$, we have $\cos 3 \varphi=1$ which implies $\left.\varphi\right|_{u=u_{0}}=0$. As $u$ varies from 0 to $u_{0}$, therefore, $\varphi$ varies from 0 to $\pi / 3-\sqrt{3 / 8} \chi$.

To see the behaviors of the level-crossing, it is useful to plot the normalized eigenvalues $t_{j} \equiv \tilde{E}_{j} / 2 R(j=1,2,3)$ of $\mathcal{M}$, instead of $\tilde{E}_{j}$ themselves. The values of $t_{j}(j=1,2,3)$ are shown together with $\cos 3 \varphi$ in Fig. [1 for $D=2.9$ as functions of $u \equiv 3 \Lambda^{2} / \Delta E_{31}^{2}$. 


\section{Appendix F: The derivation of the transition probability in the case with large} magnetic moments and a magnetic field

As is explained in the main text, we discuss the contribution from one level-crossing only. By taking into account the nonadiabatic contribution, we can integrate Eq. (54):

$$
\begin{aligned}
& \Psi(L)+i \Psi^{c}(L)=\tilde{U}(L) e^{-i \Phi_{2}} W e^{-i \Phi_{1}} \tilde{U}^{-1}(0)\left(\Psi(0)+i \Psi^{c}(0)\right) \\
& \Psi(L)-i \Psi^{c}(L)=\tilde{U}^{*}(L) e^{-i \Phi_{2}} W^{*} e^{-i \Phi_{1}}\left(\tilde{U}^{*}\right)^{-1}(0)\left(\Psi(0)-i \Psi^{c}(0)\right)
\end{aligned}
$$

where $W$ stands for the transition matrix between the two energy eigenstates at the levelcrossing $t=t_{R}$, and

$$
\begin{aligned}
\Phi_{1} & \equiv \int_{0}^{t_{R}} \tilde{\mathcal{E}}(t) d t \\
\Phi_{2} & \equiv \int_{t_{R}}^{L} \tilde{\mathcal{E}}(t) d t
\end{aligned}
$$

From this we get

$$
\begin{aligned}
\Psi(L)= & \frac{1}{2}\left[\tilde{U}(L) e^{-i \Phi_{2}} W e^{-i \Phi_{1}} \tilde{U}^{-1}(0)\left(\Psi(0)+i \Psi^{c}(0)\right)\right. \\
& \left.+\tilde{U}^{*}(L) e^{-i \Phi_{2}} W^{*} e^{-i \Phi_{1}}\left(\tilde{U}^{*}\right)^{-1}(0)\left(\Psi(0)-i \Psi^{c}(0)\right)\right]
\end{aligned}
$$

Thus the probability amplitudes for the flavor transition are given by

$$
\begin{aligned}
A\left(\nu_{\alpha} \rightarrow \nu_{\beta}\right) & =\frac{1}{2}\left[\tilde{U}(L) e^{-i \Phi_{2}} W e^{-i \Phi_{1}} \tilde{U}^{-1}(0)+\tilde{U}^{*}(L) e^{-i \Phi_{2}} W^{*} e^{-i \Phi_{1}}\left(\tilde{U}^{*}\right)^{-1}(0)\right]_{\beta \alpha} \\
& =\sum_{j, k}\left(e^{-i \Phi_{2}}\right)_{j j}\left(e^{-i \Phi_{1}}\right)_{k k} \operatorname{Re}\left\{\tilde{U}_{\beta j}(L) W_{j k} \tilde{U}_{\alpha k}^{*}(0)\right\} \\
A\left(\nu_{\alpha} \rightarrow \bar{\nu}_{\beta}\right) & =\frac{i}{2}\left[\tilde{U}(L) e^{-i \Phi_{2}} W e^{-i \Phi_{1}} \tilde{U}^{-1}(0)-\tilde{U}^{*}(L) e^{-i \Phi_{2}} W^{*} e^{-i \Phi_{1}}\left(\tilde{U}^{*}\right)^{-1}(0)\right]_{\beta \alpha} \\
& =-\sum_{j, k}\left(e^{-i \Phi_{2}}\right)_{j j}\left(e^{-i \Phi_{1}}\right)_{k k} \operatorname{Im}\left\{\tilde{U}_{\beta j}(L) W_{j k} \tilde{U}_{\alpha k}^{*}(0)\right\}
\end{aligned}
$$

Hence we obtain the transition probabilities

$$
\begin{aligned}
P\left(\nu_{\alpha} \rightarrow \nu_{\beta}\right)= & \sum_{j, k, j^{\prime}, k^{\prime}}\left(e^{-i \Phi_{2}}\right)_{j j}\left(e^{i \Phi_{2}}\right)_{j^{\prime} j^{\prime}}\left(e^{-i \Phi_{1}}\right)_{k k}\left(e^{i \Phi_{1}}\right)_{k^{\prime} k^{\prime}} \\
& \times \operatorname{Re}\left\{\tilde{U}_{\beta j}(L) W_{j k} \tilde{U}_{\alpha k}^{*}(0)\right\} \operatorname{Re}\left\{\tilde{U}_{\beta j^{\prime}}(L) W_{j^{\prime} k^{\prime}} \tilde{U}_{\alpha k^{\prime}}^{*}(0)\right\} \\
\rightarrow & \sum_{j, k}\left[\operatorname{Re}\left\{\tilde{U}_{\beta j}(L) W_{j k} \tilde{U}_{\alpha k}^{*}(0)\right\}\right]^{2} \\
P\left(\nu_{\alpha} \rightarrow \bar{\nu}_{\beta}\right) \rightarrow & \sum_{j, k}\left[\operatorname{Im}\left\{\tilde{U}_{\beta j}(L) W_{j k} \tilde{U}_{\alpha k}^{*}(0)\right\}\right]^{2}
\end{aligned}
$$

where we have taken the limit $t_{R} \rightarrow \infty, L \rightarrow \infty$ and we have averaged over rapid oscillations: $\left(e^{-i \Phi_{1}}\right)_{k k}\left(e^{i \Phi_{1}}\right)_{k^{\prime} k^{\prime}} \rightarrow \delta_{k k^{\prime}},\left(e^{-i \Phi_{2}}\right)_{j j}\left(e^{i \Phi_{2}}\right)_{j^{\prime} j^{\prime}} \rightarrow \delta_{j j^{\prime}}$. Each probability in Eqs. (F1) itself is not expressed in terms of $\tilde{X}_{j}^{\alpha \alpha}(0)$, but we find that the following relation holds:

$$
P\left(\nu_{\alpha} \rightarrow \nu_{\beta}\right)+P\left(\nu_{\alpha} \rightarrow \bar{\nu}_{\beta}\right)=\sum_{j, k}\left|\tilde{U}_{\beta j}(L)\right|^{2}\left|W_{j k}\right|^{2}\left|\tilde{U}_{\alpha k}^{*}(0)\right|^{2}
$$




$$
=\left(\begin{array}{lll}
\left|U_{\beta 1}\right|^{2} & \left|U_{\beta 2}\right|^{2} & \left|U_{\beta 3}\right|^{2}
\end{array}\right)\left(\begin{array}{ccc}
1 & 0 & 0 \\
0 & 1-P_{H} & P_{H} \\
0 & P_{H} & 1-P_{H}
\end{array}\right)\left(\begin{array}{c}
\left|\tilde{U}_{\alpha 1}(0)\right|^{2} \\
\mid \tilde{U}_{\alpha 2}(0) \\
\left|\tilde{U}_{\alpha 3}(0)\right|^{2}
\end{array}\right) \text {, }
$$

where we have assumed that the level-crossing occurs between the energy eigenstates 2 and 3 , we have used the fact that in that case $\left|W_{23}\right|^{2}=\left|W_{32}\right|^{2}=P_{H},\left|W_{22}\right|^{2}=\left|W_{33}\right|^{2}=1-P_{H}$, and we have assumed that there is no magnetic field at the endpoint $t=L$.

\section{Appendix G: The calculations of the jumping factor $P_{H}$ in the case with large magnetic moments and a magnetic field}

We have seen in Appendix E that $\varphi$ varies from 0 to $\pi / 3-\sqrt{3 / 8} \chi$, as $u$ varies from 0 to $u_{0}$. To estimate the jumping probability $P_{H}$ near the level-crossing $u=u_{0}$, let us obtain the complex solution $u$ of the equation

$$
\cos 3 \varphi=\frac{1-D u}{(1+u)^{3 / 2}}=-1
$$

for $D<3$. Eq. (G1) gives

$$
u\left\{u^{2}-\left(D^{2}-3\right) u+3+2 D\right\}=0,
$$

Thus the solutions other than $u=0$ for Eq. (G1) are

$$
u=\frac{D^{2}-3}{2} \pm i \sqrt{(3-D)(D+1)^{3}} \simeq 3 \pm 6 \sqrt{2} \chi i,
$$

where we have used the condition (E5) for small $|\chi|$. Thus we take the path of the complex integral for $P_{H}$ as

$$
u=3+6 \sqrt{2} \chi \xi i, \quad 0 \leq \xi \leq 1 .
$$

When the complex variable lies in the region specified by Eq. (G3), we have

$$
\begin{aligned}
\cos 3 \varphi & =-1+\frac{27}{16} \chi^{2}\left(1-\xi^{2}\right), \\
3 \varphi & =\pi-\sqrt{\frac{27}{8}} \chi \sqrt{1-\xi^{2}} \\
\varphi & =\frac{\pi}{3}-\sqrt{\frac{3}{8}} \chi \sqrt{1-\xi^{2}}
\end{aligned}
$$

From Eq. (E2) the difference $\Delta \tilde{E}_{32} \equiv \tilde{E}_{3}-\tilde{E}_{2}$ of the two eigenvalues is given

$$
\begin{aligned}
\Delta \tilde{E}_{32} & =2 \sqrt{3} R \sin \left(\frac{\pi}{3}-\varphi\right) \\
& =2 \sqrt{3} \frac{\Delta E_{31}}{3} \sqrt{1+u} \sin \left(\frac{\pi}{3}-\varphi\right) \\
& \simeq \sqrt{2} \Delta E_{31} \chi \sqrt{1-\xi^{2}},
\end{aligned}
$$


where we have used Eq. (G4) and the fact that $|\chi| \ll 1$ in the second line. Thus the jumping probability near the point $u=u_{0}$ is given by

$$
\begin{aligned}
P_{H} & =\exp \left[-\operatorname{Im}\left(\int_{\xi=0}^{\xi=1} \Delta \tilde{E}_{32}(t) d t\right)\right] \\
& \simeq \exp \left[-\operatorname{Im}\left(\int_{\xi=0}^{\xi=1} \sqrt{2} \Delta E_{31} \chi \sqrt{1-\xi^{2}} \frac{1}{|d \Lambda / d t|} d \Lambda\right)\right] \\
& \simeq \exp \left[-2 \Delta E_{31}^{2} \chi^{2} \operatorname{Im}\left(\int_{0}^{1} \frac{\sqrt{1-\xi^{2}}}{|d \Lambda / d t|} i d \xi\right)\right] \\
& =\exp \left[-2 \Delta E_{31}^{2} \chi^{2} \int_{0}^{1} \frac{\sqrt{1-\xi^{2}}}{|d \Lambda / d t|} d \xi\right]
\end{aligned}
$$

where we have used the fact $d \Lambda=d\left(\Delta E_{31} \sqrt{u / 3}\right)=\left(\Delta E_{31} / \sqrt{3}\right) d u /(2 \sqrt{u}) \simeq\left(\Delta E_{31} / 6\right) d u$ $=6 \sqrt{2} \Delta E_{31} \chi i d \xi$. For simplicity we assume that $|d \Lambda / d t|=$ constant, and we obtain Eq. (62).

\section{Appendix H: The calculations of the effective mixing angle and its derivative in} the case with large magnetic moments and a magnetic field

To evaluate the derivative of $\tilde{\psi}_{23}$ we need to calculate not only the derivative of $\left\{(\mathcal{M})^{j}\right\}_{\tau \tau}$ which appear in the KTY formula (7) but also the derivative of the eigenvalues $\tilde{E}_{j}$. The eigenvalues $\tilde{E}_{j}(j=1,2,3)$ in Eq. (E2) are written as

$$
\tilde{E}_{j}=2 R \cos \left(\varphi+\frac{2 j}{3} \pi\right)=\frac{\Delta E_{31}}{3} \sqrt{1+u} \cos \left(\varphi+\frac{2 j}{3} \pi\right)
$$

and, as we will see later, the derivative of $\varphi$ with respect to $u$ vanishes at the level-crossing $u=u_{0}$. To simplify the calculations, therefore, we introduce the normalized eigenvalues:

$$
\tilde{e}_{j} \equiv \frac{\tilde{E}_{j}}{R}=2 \cos \left(\varphi+\frac{2 j}{3} \pi\right)
$$

The normalized eigenvalues $\tilde{e}_{j}$ are convenient when we compute the derivative of $\tilde{\psi}_{23}$, because the derivative of $\tilde{e}_{j}$ with respect to $u$ vanishes at the level-crossing $u=u_{0}$. So we express the quantities, which are necessary to obtain $\tilde{\psi}_{23}$, in terms of $\tilde{e}_{j}$ :

$$
\begin{aligned}
\tilde{X}_{2}^{\tau \tau} & =\frac{1}{\Delta \tilde{e}_{32} \Delta \tilde{e}_{12}}\left\{\tilde{e}_{3} \tilde{e}_{1}-\left(\tilde{e}_{3}+\tilde{e}_{1}\right) y_{2}^{\tau \tau}+y_{3}^{\tau \tau}\right\}, \\
\tilde{X}_{3}^{\tau \tau} & =\frac{1}{\Delta \tilde{e}_{31} \Delta \tilde{e}_{32}}\left\{\tilde{e}_{1} \tilde{e}_{2}-\left(\tilde{e}_{1}+\tilde{e}_{2}\right) y_{2}^{\tau \tau}+y_{3}^{\tau \tau}\right\}, \\
\tan ^{2} \tilde{\psi}_{23}=\frac{\tilde{X}_{2}^{\tau \tau}}{\tilde{X}_{3}^{\tau \tau}} & =\frac{\Delta \tilde{e}_{31}}{\Delta \tilde{e}_{12}} \cdot \frac{\tilde{e}_{3} \tilde{e}_{1}-\left(\tilde{e}_{3}+\tilde{e}_{1}\right) y_{2}^{\tau \tau}+y_{3}^{\tau \tau}}{\tilde{e}_{1} \tilde{e}_{2}-\left(\tilde{e}_{1}+\tilde{e}_{2}\right) y_{2}^{\tau \tau}+y_{3}^{\tau \tau}}
\end{aligned}
$$

where we have defined

$$
\begin{aligned}
\Delta \tilde{e}_{j k} & \equiv \tilde{e}_{j}-\tilde{e}_{k}, \\
y_{j}^{\tau \tau} & \equiv \frac{\left(\mathcal{M}^{j-1}\right)_{\tau \tau}}{R^{j-1}} \cdot(j=2,3)
\end{aligned}
$$


The matrix elements of $\mathcal{M}$ and $\mathcal{M}^{2}$ can be calculated from Eq. (60) as follows:

$$
\begin{aligned}
\mathcal{M} & =\left(\begin{array}{ccc}
-\frac{1}{3} \Delta E_{31} & -i \Lambda \cos \chi & 0 \\
i \Lambda \cos \chi & -\frac{1}{3} \Delta E_{31} & -i \Lambda \sin \chi \\
0 & i \Lambda \sin \chi & \frac{2}{3} \Delta E_{31}
\end{array}\right) \\
\mathcal{M}^{2} & =\left(\begin{array}{ccc}
\frac{1}{9} \Delta E_{31}^{2}+\Lambda^{2} \cos ^{2} \chi & \frac{2}{3} i \Lambda \Delta E_{31} \cos \chi & -\frac{1}{2} \Lambda^{2} \sin 2 \chi \\
-\frac{2}{3} i \Lambda \Delta E_{31} \cos \chi & \frac{1}{9} \Delta E_{31}^{2}+\Lambda^{2} & -\frac{i}{3} \Lambda \Delta E_{31} \sin \chi \\
-\frac{1}{2} \Lambda^{2} \sin 2 \chi & \frac{i}{3} \Lambda \Delta E_{31} \sin \chi & \frac{4}{9} \Delta E_{31}^{2}+\Lambda^{2} \sin ^{2} \chi
\end{array}\right)
\end{aligned}
$$

First of all, let us evaluate the value of $\tilde{\psi}_{23}$ itself at the level-crossing $u=u_{0}$. At the level-crossing, from Eqs. (E6), (E7) and (피), we get

$$
\begin{aligned}
&\left.\tilde{e}_{1}\right|_{u=u_{0}} \simeq-2 \\
&\left.\tilde{e}_{2}\right|_{u=u_{0}} \simeq 1+\frac{3}{2 \sqrt{2}} \chi \\
&\left.\tilde{e}_{3}\right|_{u=u_{0}} \simeq 1-\frac{3}{2 \sqrt{2}} \chi \\
&\left.y_{2}^{\tau \tau}\right|_{u=u_{0}}=\left.\frac{\mathcal{M}_{\tau \tau}}{R}\right|_{u=u_{0}}=\left.\frac{(2 / 3) \Delta E_{31}}{\sqrt{\Delta E_{31}^{2} / 9+\Lambda^{2} / 3}}\right|_{u=u_{0}}=\left.\frac{2}{\sqrt{1+u}}\right|_{u=u_{0}} \simeq 1 \\
&\left.y_{3}^{\tau \tau}\right|_{u=u_{0}}=\left.\frac{\left(\mathcal{M}^{2}\right)_{\tau \tau}}{R^{2}}\right|_{u=u_{0}}=\left.\frac{(4 / 9) \Delta E_{31}^{2}+\Lambda^{2} \sin ^{2} \chi}{\Delta E_{31}^{2} / 9+\Lambda^{2} / 3}\right|_{u=u_{0}}=\left.\frac{4+3 u \sin ^{2} \chi}{1+u}\right|_{u=u_{0}} \simeq 1,
\end{aligned}
$$

where we have used the fact

$$
R=\sqrt{\frac{\Delta E_{31}^{2}}{9}+\frac{\Lambda^{2}}{3}}=\frac{\Delta E_{31}}{3} \sqrt{1+u},
$$

and we have ignored terms of order $O\left(\chi^{2}\right)$. Thus we observe that the effective mixing angle $\tilde{\psi}_{23}$ is maximal at the level-crossing:

$$
\left.\tan ^{2} \tilde{\psi}_{23}\right|_{u=u_{0}} \simeq \frac{3}{-3} \cdot \frac{-2\left(1-\frac{3}{2 \sqrt{2}} \chi\right)+1+\frac{3}{2 \sqrt{2}} \chi+1}{-2\left(1+\frac{3}{2 \sqrt{2}} \chi\right)+1-\frac{3}{2 \sqrt{2}} \chi+1} \simeq 1
$$

Hence $\tilde{\psi}_{23}$ is the appropriate effective mixing angle to describe the jumping factor $P_{H}$ as in the standard two flavor case.

Next, let us evaluate the derivative of $\tilde{\psi}_{23}$. Since the derivative of $\varphi$ at the level-crossing vanishes

$$
\begin{aligned}
\left.\frac{d \varphi}{d t}\right|_{u=u_{0}} & =\left.\frac{d u}{d t} \frac{d \varphi}{d u}\right|_{u=u_{0}} \\
& =\left.\frac{d u}{d t} \frac{d}{d u}\left[\frac{1}{3} \cos ^{-1}\left\{\frac{1-D u}{(1+u)^{3 / 2}}\right\}\right]\right|_{u=u_{0}} \\
& =\left.\frac{d u}{d t} \frac{-1}{3}\left\{1-\frac{(1-D u)^{2}}{(1+u)^{3}}\right\}^{-1 / 2} \frac{D\left(u-u_{0}\right)}{2(1+u)^{5 / 2}}\right|_{u=u_{0}}=0
\end{aligned}
$$


the derivative of $\tilde{e}_{j}=2 \cos (\varphi+2 j \pi / 3)$ at the level-crossing vanishes. So the only terms which do not vanish upon evaluating a derivative at the level-crossing $u=u_{0}$ in Eq. (H2) are $y_{2}^{\tau \tau}$ and $y_{3}^{\tau \tau}$. By taking the derivative of the logarithm of the both hand sides of Eq. ( $\mathrm{H2}$ ), we get at the level-crossing

$$
\begin{aligned}
\left.\left(2 \frac{d \tilde{\psi}_{23}}{d u} \cdot \frac{2}{\sin 2 \tilde{\psi}_{23}}\right)\right|_{u=u_{0}}= & \left.\left\{\frac{-\left(\tilde{e}_{3}+\tilde{e}_{1}\right) \frac{d y_{2}^{\tau \tau}}{d u}+\frac{d y_{3}^{\tau \tau}}{d u}}{\tilde{e}_{3} \tilde{e}_{1}-\left(\tilde{e}_{3}+\tilde{e}_{1}\right) y_{2}^{\tau \tau}+y_{3}^{\tau \tau}}-\frac{-\left(\tilde{e}_{1}+\tilde{e}_{2}\right) \frac{d y_{2}^{\tau \tau}}{d u}+\frac{d y_{3}^{\tau \tau}}{d u}}{\tilde{e}_{1} \tilde{e}_{2}-\left(\tilde{e}_{1}+\tilde{e}_{2}\right) y_{2}^{\tau \tau}+y_{3}^{\tau \tau}}\right\}\right|_{u=u_{0}} \\
= & {\left[\frac{\Delta \tilde{e}_{32}}{\left\{\tilde{e}_{3} \tilde{e}_{1}-\left(\tilde{e}_{3}+\tilde{e}_{1}\right) y_{2}^{\tau \tau}+y_{3}^{\tau \tau}\right\}\left\{\tilde{e}_{1} \tilde{e}_{2}-\left(\tilde{e}_{1}+\tilde{e}_{2}\right) y_{2}^{\tau \tau}+y_{3}^{\tau \tau}\right\}}\right.} \\
& \left.\times\left\{\frac{d y_{2}^{\tau \tau}}{d u}\left(\tilde{e}_{1}^{2}-y_{3}^{\tau \tau}\right)+\frac{d y_{3}^{\tau \tau}}{d u}\left(y_{2}^{\tau \tau}-\tilde{e}_{1}\right)\right\}\right]\left.\right|_{u=u_{0}} .
\end{aligned}
$$

Here we note

$$
\begin{aligned}
\left.\frac{d y_{2}^{\tau \tau}}{d u}\right|_{u=u_{0}} & =\left.\frac{d}{d u}\left(\frac{3}{\Delta E_{31} \sqrt{1+u}} \frac{2}{3} \Delta E_{31}\right)\right|_{u=u_{0}} \\
& =-\left.\frac{1}{(1+u)^{3 / 2}}\right|_{u=u_{0}} \simeq-\frac{1}{8} \\
\frac{d y_{3}^{\tau \tau}}{d u} & =\left.\frac{d}{d u}\left\{\frac{9}{\Delta E_{31}^{2}(1+u)}\left(\frac{4}{9} \Delta E_{31}^{2}+\frac{u}{3} \Delta E_{31}^{2} \sin ^{2} \chi\right)\right\}\right|_{u=u_{0}} \\
& \simeq-\left.\frac{4}{(1+u)^{2}}\right|_{u=u_{0}} \simeq-\frac{1}{4}
\end{aligned}
$$

where we have ignored terms of order $O\left(\chi^{2}\right)$. Taking into account $\sin 2 \tilde{\psi}_{23} \simeq 1$, Eq. (H3) gives

$$
\left.\frac{d \tilde{\psi}_{23}}{d u}\right|_{u=u_{0}}=\frac{1}{4} \frac{3 \chi / \sqrt{2}}{(-9 \chi / 2 \sqrt{2})(9 \chi / 2 \sqrt{2})}\left\{-\frac{1}{8}\left(2^{2}-1\right)-\frac{1}{4}(1+2)\right\}=\frac{1}{12 \sqrt{2} \chi} .
$$

Assuming $|d \Lambda / d t|_{u=u_{0}}=$ constant, we have

$$
\left.\frac{d \tilde{\psi}_{23}}{d t}\right|_{u=u_{0}}=\left.\left.\frac{d u}{d t}\right|_{u=u_{0}} \cdot \frac{d \tilde{\psi}_{23}}{d u}\right|_{u=u_{0}}=\frac{|d \Lambda / d t|_{u=u_{0}}}{\Delta E_{31}} \frac{1}{2 \sqrt{2} \chi}
$$

On the other hand,

$$
\left.\Delta \tilde{E}_{32}\right|_{u=u_{0}}=\left.2 \sqrt{3} R \sin \left(\frac{\pi}{3}-\varphi\right)\right|_{u=u_{0}} \simeq \sqrt{2} \Delta E_{31} \chi
$$

We conclude, therefore, that the exponent of the jumping factor coincides with $-\pi / 2$ times the $\gamma$ factor in the case of a linear potential $(F=1)$ :

$$
\gamma=\left.\frac{\Delta \tilde{E}_{32}}{2\left|d \tilde{\psi}_{23} / d t\right|}\right|_{u=u_{0}} \simeq \frac{2 \Delta E_{31}^{2} \chi^{2}}{|d \Lambda / d t|_{u=u_{0}}}=-\frac{\log P_{H}}{\pi / 2}
$$




\section{Acknowledgments}

The author would like to thank Akira Shudo for discussions on the Landau-Zener theory in various stages of this work. This research was partly supported by a Grant-in-Aid for Scientific Research of the Ministry of Education, Science and Culture, under Grant No. 24540281 and No. 25105009.

[1] Y. Fukuda et al. [Super-Kamiokande Collaboration], Phys. Rev. Lett. 81, 1562 (1998) arXiv:hep-ex/9807003.

[2] K. Kimura, A. Takamura and H. Yokomakura, Phys. Lett. B 537, 86 (2002) arXiv:hep-ph/0203099.

[3] K. Kimura, A. Takamura and H. Yokomakura, Phys. Rev. D 66, 073005 (2002) arXiv:hep-ph/0205295.

[4] H. Zhang, Mod. Phys. Lett. A 22, 1341 (2007) arXiv:hep-ph/0606040.

[5] O. Yasuda, arXiv:0704.1531 [hep-ph].

[6] L. Landau, Phys. Z. Sowj. 2, 46 (1932).

[7] C. Zener, Proc. Roy. Soc. Lond. A 137, 696 (1932).

[8] T. K. Kuo and J. T. Pantaleone, Rev. Mod. Phys. 61, 937 (1989).

[9] K. Yamamoto, arXiv:1003.2853 [hep-ph].

[10] J. T. Pantaleone, Phys. Lett. B 287, 128 (1992).

[11] S. Samuel, Phys. Rev. D 48, 1462 (1993).

[12] A. S. Dighe and A. Y. Smirnov, Phys. Rev. D 62, 033007 (2000) hep-ph/9907423.

[13] Z. z. Xing and H. Zhang, Phys. Lett. B 618, 131 (2005) arXiv:hep-ph/0503118.

[14] H. W. Zaglauer and K. H. Schwarzer, Z. Phys. C 40, 273 (1988).

[15] W. Grimus and T. Scharnagl, Mod. Phys. Lett. A 8, 1943 (1993).

[16] T. -K. Kuo and J. T. Pantaleone, Phys. Rev. D 39, 1930 (1989).

[17] L. Wolfenstein, Phys. Rev. D 17, 2369 (1978).

[18] M. M. Guzzo, A. Masiero and S. T. Petcov, Phys. Lett. B 260, 154 (1991);

[19] E. Roulet, Phys. Rev. D 44, 935 (1991).

[20] S. Davidson, C. Pena-Garay, N. Rius and A. Santamaria, JHEP 0303, 011 (2003) arXiv:hep-ph/0302093.

[21] A. Friedland and C. Lunardini, Phys. Rev. D 72, 053009 (2005) arXiv:hep-ph/0506143.

[22] A. Friedland, C. Lunardini and C. Pena-Garay, Phys. Lett. B 594, 347 (2004) hep-ph/0402266.

[23] A. Cisneros, Astrophys. Space Sci. 10, 87 (1971).

[24] L. B. Okun, M. B. Voloshin and M. I. Vysotsky, Sov. Phys. JETP 64, 446 (1986) [Zh. Eksp. Teor. Fiz. 91, 754 (1986)].

[25] C. -S. Lim and W. J. Marciano, Phys. Rev. D 37, 1368 (1988).

[26] E. K. Akhmedov, Phys. Lett. B 213, 64 (1988). 